\title{
Gravitational-wave amplitudes for compact binaries in eccentric orbits at the third post-Newtonian order: Memory contributions
}

Ebersold, Michael ; Boetzel, Yannick ; Faye, Guillaume ; Mishra, Chandra Kant ; Iyer, Bala R ; Jetzer, Philippe

\begin{abstract}
We compute the nonlinear memory contributions to the gravitational-wave amplitudes for compact binaries in eccentric orbits at the third post-Newtonian (3PN) order in general relativity. These contributions are hereditary in nature as they are sourced by gravitational waves emitted during the binary's entire dynamical past. Combining these with already available instantaneous and tail contributions, we get the complete $3 \mathrm{PN}$ accurate gravitational waveform.
\end{abstract}

DOI: https://doi.org/10.1103/physrevd.100.084043

Posted at the Zurich Open Repository and Archive, University of Zurich

ZORA URL: https://doi.org/10.5167/uzh-175966

Journal Article

Published Version

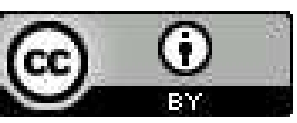

The following work is licensed under a Creative Commons: Attribution 4.0 International (CC BY 4.0) License.

Originally published at:

Ebersold, Michael; Boetzel, Yannick; Faye, Guillaume; Mishra, Chandra Kant; Iyer, Bala R; Jetzer, Philippe (2019). Gravitational-wave amplitudes for compact binaries in eccentric orbits at the third post-Newtonian order: Memory contributions. Physical review D, 100(8):084043.

DOI: https://doi.org/10.1103/physrevd.100.084043 


\title{
Gravitational-wave amplitudes for compact binaries in eccentric orbits at the third post-Newtonian order: Memory contributions
}

\author{
Michael Ebersold $\odot,{ }^{1}$ Yannick Boetzel, ${ }^{1}$ Guillaume Faye, ${ }^{2}$ Chandra Kant Mishra, ${ }^{3}$ Bala R. Iyer, ${ }^{4}$ and Philippe Jetzer ${ }^{1}$ \\ ${ }^{1}$ Physik-Institut, Universität Zürich, Winterthurerstrasse 190, 8057 Zürich \\ ${ }^{2} \mathscr{G} \mathbb{R} \mathbb{C O}$, Institut d'Astrophysique de Paris, UMR 7095, CNRS, Sorbonne Université, \\ $98^{\text {bis }}$ boulevard Arago, 75014 Paris, France \\ ${ }^{3}$ Department of Physics, Indian Institute of Technology, Madras, Chennai 600036, India \\ ${ }^{4}$ International Centre for Theoretical Sciences, Tata Institute of Fundamental Research, \\ Bangalore 560089, India
}

(Received 14 June 2019; published 21 October 2019)

\begin{abstract}
We compute the nonlinear memory contributions to the gravitational-wave amplitudes for compact binaries in eccentric orbits at the third post-Newtonian (3PN) order in general relativity. These contributions are hereditary in nature as they are sourced by gravitational waves emitted during the binary's entire dynamical past. Combining these with already available instantaneous and tail contributions, we get the complete 3PN accurate gravitational waveform.
\end{abstract}

DOI: $10.1103 /$ PhysRevD.100.084043

\section{INTRODUCTION}

The observation of the first gravitational-wave $(\mathrm{GW})$ signal by LIGO and Virgo opened up the new field of gravitational-wave astronomy [1-4]. So far, ten confirmed binary black hole mergers and one binary neutron star coalescence have been reported [5-10]. KAGRA [11] is expected to join the global network of detectors later this year, followed by LIGO-India in 2025 [12], leading to improved parameter estimation and source localization. These ground-based detectors are sensitive to the decahertz-kilohertz frequency of the GW spectrum. In the future, the space-based detector LISA [13] will probe lower frequencies (around the millihertz range), and pulsar timing arrays (PTAs) may measure ultralow (nanohertz) frequency GWs [14].

Currently (and this will most probably not change in the future), compact binaries are the most important sources of observable GW signals. The events detected so far have all been found using circular templates. However, we know that binaries with substantial eccentricity exist, e.g., the Hulse-Taylor binary with an eccentricity of $e \sim 0.6$ [15]. Nonetheless, at the time this binary enters the detection band of ground-based GW detectors, it will have circularized to a negligible $e \sim 10^{-5}$ and not be distinguishable from a circular binary with current detector sensitivity [1618]. In particular, in globular clusters and galactic nuclei there are expected to be binaries with non-negligible eccentricity $(e>0.1)$ emitting detectable GWs [19-24]. Hence, the detection of GWs from eccentric compact binaries could provide important information on compact object populations in globular clusters and galactic nuclei [25].

As soon as LISA is operational, it will be able to observe compact binaries in our galaxy emitting GWs of much lower frequency. At this point they still are expected to have moderate eccentricities [26,27]. On the other hand, LISA should be able to detect supermassive black hole binaries forming in the aftermath of galaxy mergers. Notably, triple-induced coalescences are expected to have large eccentricities that remain significant until merger [28-32].

The above anticipated prospects of future GW observations have motivated the development of eccentric waveform models. In the inspiraling phase one usually uses the post-Newtonian (PN) formalism to model the dynamics of the binary. This introduces three distinct time scales. The first two, the orbital and periastron precession time scales, are associated with the conservative dynamics and commonly described by the quasi-Keplerian parametrization [33,34]. The third time scale appears when the dissipative radiation-reaction effects are taken into account $[35,36]$. Several waveform models have been built using this description of a binary [18,37-46]. In general, the farzone gravitational radiation field receives instantaneous and hereditary contributions. The instantaneous part is determined by the state of its source at a given retarded time, while the hereditary part depends on the entire dynamical history of the source. In particular, the latter contains tail and memory pieces.

In this work, we concentrate on the memory contributions to the waveform from eccentric binaries. Normally we think of gravitational waves as oscillatory perturbations 
propagating on the background metric at the speed of light. However, all GW sources are subject to the so-called gravitational-wave memory effect, which manifests in a difference of the observed GW amplitudes at late and early times:

$$
\Delta h_{\mathrm{mem}}=\lim _{t \rightarrow+\infty} h(t)-\lim _{t \rightarrow-\infty} h(t) .
$$

In an ideal, freely falling GW detector the GW memory causes a permanent displacement after the GW has passed. There are two main types of GW memory. The linear memory [47] originates from a net change in the time derivatives of the source multipole moments between early and late times, present mainly in unbound (e.g., hyperbolic binary) systems. For bound systems the linear memory is negligible, as long as the components were formed, captured, or underwent mass loss long before the GWdriven regime. The nonlinear memory, also called the "Christodoulou memory" [48-52], is a phenomenon directly related to the nonlinearity of general relativity. It arises from GWs sourced by previously emitted GWs. Since the nonlinear memory is not produced directly by the source but rather by its radiation, it is present in all sources of GWs. From a more theoretical perspective, the memory effect and its variants can be interpreted in terms of conserved charges at null infinity and "soft theorems" $[53,54]$. Several methods to look for the memory effect have been devised. PTAs would observe a sudden change in the pulse frequency of a pulsar [55-58] and ground-based detectors like LIGO-although not sensitive enough to the memory of a single event-could allow for a detection from the accumulation of several events. [59-61].

For circular binaries, the nonlinear, nonoscillatory memory contributions to the waveform were computed at the 3PN order in Ref. [62]. Regarding eccentric binaries, the leading-order zero-frequency or the so-called direct current (DC) memory terms were obtained in Ref. [63]. In this paper, we extend these computations to the 3PN level by computing all terms coming from the memory contribution to the radiative mass multipoles. Note that this yields not only the "genuine" DC memory, but also oscillatory contributions. In the circular limit, the latter have been computed in Ref. [64]. Due to complicated hereditary integrals, we calculate the memory contributions within a small-eccentricity expansion. We present all of our results in modified harmonic (MH) gauge in terms of the postNewtonian parameter $x=\left(G m \bar{\omega} / c^{3}\right)^{2 / 3}$ and the eccentricity $e=\bar{e}_{t}$, with $\bar{\omega}=(1+\bar{k}) \bar{n}$ being the orbital frequency and $\bar{n}=2 \pi / P$ the mean motion. With the instantaneous contributions already available [65], and the tail and postadiabatic contributions computed in a companion paper [66] — hereafter called Paper I-this work aims to complete the knowledge of the 3PN waveform valid during the early inspiral of eccentric binary systems.
This paper is structured as follows. In Sec. II we discuss how the nonlinear memory arises from the gravitationalwave energy flux and how it can be computed by integrating this flux over the binary's past history. In Sec. III we explicitly evaluate the past-history integrals, which lead to two types of memory terms-DC memory and oscillatory memory-that are discussed separately in Secs. III B and III C. We next combine our results with the already available instantaneous and tail contributions and discuss the full 3PN waveform in Sec. IV. In Sec. V we give a brief summary and conclude our work. Most expressions in this paper are presented only to leading order in eccentricity for convenience, though we provide the complete results to $\mathcal{O}\left(e^{6}\right)$ in a supplemental Mathematica notebook [67].

\section{PREREQUISITES}

\section{A. Memory contribution to the mass multipole moments}

Here we briefly state the essentials of the memory calculation. The conventions and notations used are the same as those outlined in Sec. II of Paper I.

The gravitational waveform polarizations can be uniquely decomposed into the spherical harmonic modes $h^{\ell m}$ via

$$
h_{+}-\mathrm{i} h_{\times}=\sum_{\ell=2}^{\infty} \sum_{m=-\ell}^{\ell} h^{\ell m} Y_{-2}^{\ell m}(\Theta, \Phi),
$$

where the basis is formed by the spin-weighted spherical harmonics $Y_{-2}^{\ell m}(\Theta, \Phi)$ and the amplitude modes

$$
h^{\ell m}=-\frac{G}{\sqrt{2} R c^{\ell+2}}\left(U^{\ell m}-\frac{\mathrm{i}}{c} V^{\ell m}\right)
$$

are given in terms of radiative mass and current multipoles, $U^{\ell m}$ and $V^{\ell m}$. These contain both instantaneous and hereditary parts. In the latter, we can further distinguish between tail and memory contributions (some of which may actually be tail induced) at the $3 \mathrm{PN}$ order by schematically writing

$$
\begin{gathered}
U^{\ell m}=U_{\text {inst }}^{\ell m}+U_{\text {tail }}^{\ell m}+U_{\text {mem }}^{\ell m}+\delta U^{\ell m}, \\
V^{\ell m}=V_{\text {inst }}^{\ell m}+V_{\text {tail }}^{\ell m}+\delta V^{\ell m},
\end{gathered}
$$

where $\delta U^{\ell m}$ and $\delta V^{\ell m}$ represent possible higher-order hereditary terms. Note that there is no memory contribution to the radiative current-type moments [51]. Now, employing the multipolar post-Minkowskian post-Newtonian (PN) formalism, the radiative moments can be written in terms of the source moments. These relations can be found in Sec. III A of Ref. [65] for the instantaneous parts, which only require the knowledge of the source motion at a given 
moment in retarded time $T_{R}$, and in Sec. II B of Paper I for the hereditary parts, which involve integrals over the entire dynamical past of the source.

The nonlinear memory may be expressed in terms of the time derivative of the gravitational waveform by solving one component of Einstein's equations near future null infinity in Bondi coordinates $[53,68]$. In this approach, the complex wave amplitude $h_{+}-\mathrm{i} h_{\times}$is decomposed into even-parity and odd-parity pieces, the former being parametrized by a scalar function of the retarded time $T_{R}$ and the angles $(\Theta, \Phi)$, namely, $\Phi_{\mathrm{e}}\left(T_{R}, \Theta, \Phi\right)=$ $\sum_{\ell \geq 0,|m| \leq \ell} \Phi_{\mathrm{e}}^{\ell m}\left(T_{R}\right) Y^{\ell m}(\Theta, \Phi)$, where the $\Phi_{\mathrm{e}}^{\ell m}\left(T_{R}\right)$ turn out to be equal to $\sqrt{2(\ell-2) ! /(\ell+2) !}$ with our conventions. The memory then manifests itself as a lowfrequency shift of those modes. Since this effect is sourced by GWs, the consequent change for $U_{\mathrm{mem}}^{\ell m}$ is a functional of the gravitational-wave "flux"

$$
\frac{\mathrm{d} E^{\mathrm{GW}}}{\mathrm{d} t \mathrm{~d} \Omega} \equiv \frac{c^{3} R^{2}}{16 \pi G}\left(\dot{h}_{+}^{2}+\dot{h}_{\times}^{2}\right) .
$$

More precisely, the nonlinear memory contribution to the radiative mass moment $U^{\ell m}\left(T_{R}\right)$ is given by [53]

$U_{\mathrm{mem}}^{\ell m}=\frac{32 \pi}{c^{2-\ell}} \sqrt{\frac{(\ell-2) !}{2(\ell+2) !}} \int_{-\infty}^{T_{R}} \mathrm{~d} t \int \mathrm{d} \Omega \frac{\mathrm{d} E^{\mathrm{GW}}}{\mathrm{d} t \mathrm{~d} \Omega} \bar{Y}^{\ell m}(\Omega)$.

This formula was first shown to hold at quadratic order in $G$ [51] before its validity was extended to the general case (see also Ref. [69], which indicates how to perturbatively construct a radiative-type gauge in which the derivation proposed in Ref. [51] can be adapted in principle to arbitrarily high orders). We will start from Eq. (6) to compute the memory contributions to the GW amplitude to the $3 \mathrm{PN}$ order.

Inserting the mode decomposition defined in Eq. (2) into Eq. (5), we find the GW energy flux in terms of the time derivatives of the $h^{\ell m}$ modes:

$$
\begin{aligned}
\frac{\mathrm{d} E^{\mathrm{GW}}}{\mathrm{d} t \mathrm{~d} \Omega}= & \frac{c^{3} R^{2}}{16 \pi G} \sum_{\ell^{\prime}=2}^{\infty} \sum_{\ell^{\prime \prime}=2}^{\infty} \sum_{m^{\prime}=-\ell^{\prime}}^{\ell^{\prime}} \sum_{m^{\prime \prime}=-\ell^{\prime \prime}}^{\ell^{\prime \prime}} \dot{h}^{\ell^{\prime} m^{\prime}} \dot{\bar{h}}^{\ell^{\prime \prime} m^{\prime \prime}} \\
& \times Y_{-2}^{\ell^{\prime} m^{\prime}}(\theta, \phi) \bar{Y}_{-2}^{\ell^{\prime \prime} m^{\prime \prime}}(\theta, \phi) .
\end{aligned}
$$

We insert this expression in turn into Eq. (6). The time derivative of the memory contribution to the mass multipole moment, $U_{\mathrm{mem}}^{\ell m(1)}=\mathrm{d} U_{\mathrm{mem}}^{\ell m} / \mathrm{d} T_{R}$, which is nothing but

\footnotetext{
${ }^{1}$ In Eq. (5), at future null infinity the product of $R^{2}$ with the term between brackets reduces to $N_{A B} N^{A B} / 2$ in the notation of Ref. [68].
}

the memory contribution before integration over past history, may thus be expressed as

$$
\begin{aligned}
U_{\mathrm{mem}}^{\ell m(1)}= & \frac{c^{\ell+1} R^{2}}{G} \sqrt{\frac{2(\ell-2) !}{(\ell+2) !}} \sum_{\ell^{\prime}=2}^{\infty} \sum_{\ell^{\prime \prime}=2}^{\infty} \sum_{m^{\prime}=-\ell^{\prime}}^{\ell^{\prime}} \sum_{m^{\prime \prime}=-\ell^{\prime \prime}}^{\ell^{\prime \prime}} \\
& \times G_{m m^{\prime} m^{\prime \prime}}^{\ell \ell^{\prime} \ell^{\prime \prime}}, \dot{\ell}^{\ell^{\prime} m^{\prime}} \dot{\bar{h}}^{\ell^{\prime \prime} m^{\prime \prime}},
\end{aligned}
$$

where $G_{m m^{\prime} m^{\prime \prime}}^{\ell \ell^{\prime} l^{\prime \prime}}$ is the angular integral of a product of three spin-weighted spherical harmonics,

$$
G_{m m^{\prime} m^{\prime \prime}}^{\ell \ell^{\prime \prime}}=\int \mathrm{d} \Omega \bar{Y}^{\ell m} Y_{-2}^{\ell^{\prime} m^{\prime}} \bar{Y}_{-2}^{\ell \ell^{\prime \prime} m^{\prime \prime}}
$$

Reference [70] provides an explicit formula for this integral:

$$
\begin{aligned}
G_{m m^{\prime} m^{\prime \prime}}^{\ell \ell^{\prime \prime}}= & (-1)^{m+m^{\prime}} \sqrt{\frac{(2 \ell+1)\left(2 \ell^{\prime}+1\right)\left(2 \ell^{\prime \prime}+1\right)}{4 \pi}} \\
& \times\left(\begin{array}{ccc}
\ell & \ell^{\prime} & \ell^{\prime \prime} \\
0 & -2 & 2
\end{array}\right)\left(\begin{array}{ccc}
\ell & \ell^{\prime} & \ell^{\prime \prime} \\
-m & m^{\prime} & -m^{\prime \prime}
\end{array}\right) .
\end{aligned}
$$

The brackets denote the Wigner 3-j symbols.

\section{B. Instantaneous and tail parts of the spherical harmonic modes}

Remembering that the dominant modes correspond to the quadrupolar case $\ell=2$, with $h^{2 m}=\mathcal{O}\left(c^{-4}\right)$, we see from Eq. (8) that the memory integrands are of $2.5 \mathrm{PN}$ order. However, as discussed below, in addition to oscillatory complex exponentials the $U_{\mathrm{mem}}^{\ell(1)}$ also contain nonoscillatory terms. Due to the integration over the past history, their contributions at times $t \leq T_{R}$ accumulate and enhance the result by a net factor $c^{5}$. It follows that the leading memory effect in the polarizations actually arises at the relative Newtonian order. Thus, Eq. (8) implies that, as an input for the computation of the 3PN-accurate $U_{\mathrm{mem}}^{\ell m(1)}$, we need a priori all nonmemory $h^{\ell m}$ modes to 3PN order. It is in fact not surprising that part of the waveform is required to calculate the full waveform since the nonlinear memory originates from gravitational waves sourced by the energy flux of gravitational waves emitted in the past, as shown by Eq. (6). Note that the contribution from the memory to the memory itself turns out not to enter the waveform up to the 3PN order. In Ref. [62] it was argued that for circular binaries these contributions would appear at the 5PN level, though for eccentric binaries we find, by explicit calculation, that these appear already at the 4PN order. This is due to additional oscillatory memory contributions that will be discussed in Sec. III C below. However, for the present work, these memory-of-memory terms can be safely ignored. 
The instantaneous parts of the 3PN-accurate $h^{\ell m}$ modes describing inspiraling eccentric binaries have been computed in Ref. [65]. The tail contributions were derived in Paper I, as well as the post-adiabatic corrections to the instantaneous contributions. The instantaneous mode amplitudes from Ref. [65] are written in terms of the post-Newtonian parameter $x$ and time eccentricity $e_{t}$, and are parametrized by the eccentric anomaly $u$. They are valid for arbitrary eccentricities, while the tail contributions in Paper I are given in a small-eccentricity expansion, parametrized by the mean anomaly $l$. The same will hold for the memory parts. Inverting the 3PN-accurate Kepler equation by means of the solution developed in Ref. [71], the instantaneous terms can be parametrized by the mean anomaly as well. Instead of restating the quasi-Keplerian parametrization and the phasing formalism describing the dynamics of the binary, we refer the reader to Secs. II C and II D of Paper I where those aspects are summarized with the same conventions and notations.

The $h^{\ell m}$ modes including instantaneous, tail, and postadiabatic contributions are given in the following form:

$$
h^{\ell m}=\frac{8 G m \nu}{c^{2} R} x \sqrt{\frac{\pi}{5}} \mathrm{e}^{-\mathrm{i} m \psi} H^{\ell m},
$$

where the $H^{\ell m}$ are written in terms of the adiabatic postNewtonian parameter $x \equiv \bar{x}$ and time eccentricity $e \equiv \bar{e}_{t}$, and are parametrized by the angles $\xi$ and $\psi$. See, for instance, Eq. (76) of Paper I for the dominant mode $\left(h^{22}\right)$ expression. The phase angles $\xi$ and $\psi$ arise naturally when applying a certain shift to the time coordinate aimed at eliminating the arbitrary constant $x_{0}$ appearing in both the instantaneous and tail parts [72,73] through the redefinitions

$$
\begin{gathered}
\xi=l-\frac{3 G M}{c^{3}} n \ln \left(\frac{x}{x_{0}^{\prime}}\right), \\
\lambda_{\xi}=\lambda-\frac{3 G M}{c^{3}}(1+k) n \ln \left(\frac{x}{x_{0}^{\prime}}\right),
\end{gathered}
$$

where $M=m(1-\nu x / 2)$ denotes the Arnowitt-DeserMisner (ADM) mass, $m=m_{1}+m_{2}$ is the total mass, $\nu=m_{1} m_{2} / m^{2}$ is the symmetric mass ratio, and $x_{0}^{\prime}$ is related to $x_{0}$ by

$$
\ln x_{0}^{\prime}=\frac{11}{18}-\frac{2}{3} \gamma_{E}-\frac{4}{3} \ln 2+\frac{2}{3} \ln x_{0},
$$

with $\gamma_{E}$ being Euler's constant. We refer to the Appendix B of Paper I for the relations between the orbital elements $(l, \lambda, \phi)$ and their redefined counterparts $\left(\xi, \lambda_{\xi}, \psi\right)$.

\section{COMPUTATION OF THE NONLINEAR MEMORY}

\section{A. Memory contributions to the time derivative of the radiative moments}

The computation of the memory contributions to the radiative mass multipole using Eq. (8) involves products of the time derivatives of the $h^{\ell m}$ modes given in Eq. (11). These are obtained by expressing $\psi$ in terms of $\xi$ and $\lambda_{\xi}$ and applying the following time derivative operator:

$$
\frac{\mathrm{d}}{\mathrm{d} t}=n\left[\frac{\mathrm{d}}{\mathrm{d} \xi}+(1+k) \frac{\mathrm{d}}{\mathrm{d} \lambda_{\xi}}\right]+\frac{\mathrm{d} x}{\mathrm{~d} t} \frac{\mathrm{d}}{\mathrm{d} x}+\frac{\mathrm{d} e}{\mathrm{~d} t} \frac{\mathrm{d}}{\mathrm{d} e},
$$

where we have used the facts that $\mathrm{d} \xi / \mathrm{d} t=\mathrm{d} l / \mathrm{d} t=n$ and $\mathrm{d} \lambda_{\xi} / \mathrm{d} t=\mathrm{d} \lambda / \mathrm{d} t=(1+k) n$ to the required PN order. The secular time evolution of $x$ and $e$ is given, at leading order, by the formulas of Peters and Mathews [16,74]

$$
\begin{array}{r}
\frac{\mathrm{d} x}{\mathrm{~d} t}=\frac{c^{3} \nu}{G m} \frac{x^{5}}{\left(1-e^{2}\right)^{7 / 2}}\left(\frac{64}{5}+\frac{584}{15} e^{2}+\frac{74}{15} e^{4}\right), \\
\frac{\mathrm{d} e}{\mathrm{~d} t}=-\frac{c^{3} \nu}{G m} \frac{e x^{4}}{\left(1-e^{2}\right)^{5 / 2}}\left(\frac{304}{15}+\frac{121}{15} e^{2}\right) .
\end{array}
$$

Note that they cause a 2.5PN correction, and thus the leading order is sufficient here. When computing the time derivatives of the amplitude modes $h^{\ell m} \sim x^{\ell / 2} / c^{2}$, we have the following leading-order PN scaling:

$$
\dot{h}^{\ell m} \sim\left(\omega / c^{2}\right) x^{\ell / 2} \sim c x^{\ell / 2+3 / 2} .
$$

As the dominant mode $\ell=2$ is of order $c x^{5 / 2}$, the knowledge of the waveform to $3 \mathrm{PN}$ order requires modes up to $\ell=8$. According to this argument, the sums in Eq. (8) consisting of products $\dot{h}^{\ell^{\prime} m^{\prime}} \dot{\bar{h}}^{\ell^{\prime \prime} m^{\prime \prime}}$ may be truncated at $\ell^{\prime}=\ell^{\prime \prime}=8$. Moreover, the appearance of the $3-j$ symbols in Eq. (10) imply some selection rules: the three lower entries have to add up to zero, i.e., $m=m^{\prime}-m^{\prime \prime}$. Since the mode products appearing in Eq. (8) scale like

$$
\dot{h}^{\ell^{\prime} m^{\prime}} \dot{\bar{h}}^{\ell \prime m^{\prime \prime}} \sim x^{n / 2} \mathrm{e}^{-\mathrm{i}\left(m^{\prime}-m^{\prime \prime}\right) \lambda_{\xi}},
$$

for some integer $n$, only memory modes with $m=0$ will contain DC terms, as was previously found for circular orbits [62]. The scaling being the same as in that case, we have to compute the $U_{\mathrm{mem}}^{\ell-(1)}$ up to $\ell=10$. On the other hand, a mode separation property holds for planar orbits [75,76]: the $h^{\ell m}$ only depend on the mass (current) radiative moments if $\ell+m$ is even (odd). Thus, as there is no memory effect in the current radiative moment, there is no memory effect when $\ell+m$ is odd. 
As an example, we show the leading-order part of the 20-mode up to $\mathcal{O}\left(e^{2}\right)$, which will represent the dominant memory contribution:

$$
\begin{aligned}
U_{\mathrm{mem}}^{20(1)}= & -\sqrt{\frac{\pi}{15}} \frac{c^{5} \nu^{2}}{G} x^{5}\left(\frac{256}{7}+\frac{5008 e^{2}}{21}+\frac{768}{7} e \mathrm{e}^{-\mathrm{i} \xi}\right. \\
& \left.+\frac{768}{7} e \mathrm{e}^{\mathrm{i} \xi}+\frac{5176}{21} e^{2} \mathrm{e}^{-2 \mathrm{i} \xi}+\frac{5176}{21} e^{2} \mathrm{e}^{2 \mathrm{i} \xi}\right) .
\end{aligned}
$$

We observe two different type of terms: oscillatory terms proportional to $\mathrm{e}^{-\mathrm{i} m^{\prime} \xi}$, and nonoscillatory ones, which give rise to the well-known leading-order DC memory.

As argued above, the $m \neq 0$ modes only contain oscillatory terms since they are proportional to $\mathrm{e}^{-\mathrm{i} m \lambda_{\xi}}$. For instance, the leading order of the 22-mode explicitly is

$$
\begin{aligned}
U_{\text {mem }}^{22(1)}= & -\sqrt{\frac{2 \pi}{5}} \frac{c^{5} \nu^{2}}{G} x^{5} \mathrm{e}^{-2 \mathrm{i} \lambda_{\xi}}\left(\frac{40}{7} e^{2}-\frac{32}{21} e \mathrm{e}^{-\mathrm{i} \xi}\right. \\
& \left.+\frac{32}{21} e \mathrm{e}^{\mathrm{i} \xi}-\frac{172}{21} e^{2} \mathrm{e}^{-2 \mathrm{i} \xi}+\frac{52}{21} e^{2} \mathrm{e}^{2 \mathrm{i} \xi}\right) .
\end{aligned}
$$

However, in Eq. (8) (used for the calculation of the $U_{\mathrm{mem}}^{\ell m(1)}$ ) there is no need to average the mode products $\dot{h}^{\ell^{\prime} m^{\prime}} \dot{h}^{\ell^{\prime \prime} m^{\prime \prime}}$ over several wavelengths. Other derivations of the memory effect, making use of the Isaacson gravitational-wave stress-energy tensor [77], resort to such a procedure. In Ref. [63], which follows this approach, the orbital average entering the calculation of the leading-order eccentric memory effectively removes the terms proportional to $\mathrm{e}^{\mathrm{i} m^{\prime} \xi}$ in the $U_{\mathrm{mem}}^{\ell 0(1)}$, so that only the terms yielding the DC memory are left over, while the discarded pieces do not affect the amplitude of the DC memory. In the absence of the orbital average, these pieces lead to small-amplitude oscillatory contributions to the waveform, which here we will call oscillatory memory contributions. It would actually be difficult to introduce an orbital average in the $m \neq 0$ modes because these terms oscillate not only on the orbital time scale, but also on the much longer precession time scale.

\section{B. DC memory}

The next step consists in evaluating the hereditary time integral

$$
U_{\mathrm{mem}}^{\ell m}=\int_{-\infty}^{T_{R}} \mathrm{~d} t U_{\mathrm{mem}}^{\ell m(1)} .
$$

To do so, we need a model for the secular evolution of the binary undergoing gravitational radiation-reaction forces. The secular 3PN-order evolution equations of the orbital elements for a quasielliptical, inspiraling binary were obtained in Refs. [78-80]. This model is an idealization since it assumes that the two components start at infinite separation and the orbital energy decreases solely due to the emission of gravitational waves.

The explicit integrals appearing in Eq. (20) are of two different types. The first one consists of a product of $x$ and $e$, each with some power $p$ and $q$, respectively:

$$
U_{\mathrm{DC}}^{\ell 0} \sim \int_{-\infty}^{T_{R}} \mathrm{~d} t x^{p}(t) e^{q}(t) .
$$

The leading Newtonian order corresponds to $p=5$. The possible values of the integer $q$ range from 0 - the quasicircular limit - to the order of the eccentricity expansion. These integrals give the nonoscillatory contributions to the waveform, i.e., the DC memory. Note that, as argued above, these terms are only present in the $m=0$ modes. The second type of integrals will lead to oscillatory terms appearing at $1.5 \mathrm{PN}, 2.5 \mathrm{PN}$, and $3 \mathrm{PN}$ order in the waveform. We will discuss these in Sec. III C.

The strategy to evaluate the integral in Eq. (21) is to express the PN parameter $x$ in terms of the eccentricity $e$ and change the integration variable from time $t$ to $e$, so that the integral runs from some initial eccentricity $e_{i}$ at early times to $e\left(T_{R}\right)$ at the current retarded time:

$$
U_{\mathrm{DC}}^{\ell 0} \sim \int_{e_{i}}^{e\left(T_{R}\right)} \mathrm{d} e\left(\frac{\mathrm{d} e}{\mathrm{~d} t}\right)^{-1} x^{p}(e) e^{q} .
$$

The time evolution of $x$ and $e$ due to radiation reaction is stated to leading order in Eqs. (15a)-(15b). Here, we need the evolution equations up to 3PN order, which are provided in Appendix B. We form the ratio of the two equations, thereby canceling the time dependence, and expand the right-hand side in $x$ and $e$. This yields a differential equation with the following structure:

$$
\begin{aligned}
\frac{\mathrm{d} x}{\mathrm{~d} e}= & f_{\mathrm{N}}(e) x+f_{1}(e) x^{2}+f_{1.5}(e) x^{5 / 2} \\
& +f_{2}(e) x^{3}+f_{2.5}(e) x^{7 / 2}+f_{3}(e, \ln x) x^{4} .
\end{aligned}
$$

Here, the $f_{i}(e)$ terms represent the coefficients of $x^{i+1}$ in the expansion of $\mathrm{d} x / \mathrm{d} e$, with $f_{\mathrm{N}}=f_{0}$. To solve this differential equation, we search for the unknown function $x(e)$ in the form of a perturbative expansion, according to

$x=x_{\mathrm{N}}+\epsilon x_{1}+\epsilon^{3 / 2} x_{1.5}+\epsilon^{2} x_{2}+\epsilon^{5 / 2} x_{2.5}+\epsilon^{3} x_{3}$,

where $\epsilon$ is a formal parameter that allows one to keep track of the PN order. Inserting this expansion into Eq. (23) and identifying the coefficients of $\epsilon^{i}$ on the left- and right-hand sides of the resulting equation, we find the set of differential relations satisfied by the post-Newtonian orders of $x$. This system can be straightforwardly solved by quadrature. Putting the pieces together yields the PN parameter $x$ as a function of eccentricity. At leading order in the PN and eccentricity expansion, we recover [35] 


$$
x(e)=x_{0}\left(\frac{e_{0}}{e}\right)^{12 / 19}
$$

where $x_{0}$ is the value of $x$ at some reference eccentricity $e_{0}$. The full 3PN result to leading order in eccentricity is provided in Appendix B. Note that for the expansion in eccentricity to be valid, the eccentricity has to be small at all times, and hence $e_{0}$ has to be small as well.

We are now in the position to insert the evolution equation for $e$ and the solution for $x(e)$ into Eq. (22). Expanding again in $x$ and $e$ yields elementary integrals, which must be calculated. We then reexpress this result in terms of the timedependent quantities $x$ and $e$ by solving their relation [Eqs. (25) and (B8)] for $x_{0}$ and reinsert the expression of this quantity in terms of $x$ and $e$ into the calculated memory terms. A final Taylor expansion then yields the DC memory pieces of the mass multipole moments.

We present the memory contributions to the spherical harmonic modes in the following form:

$$
\begin{aligned}
h_{\mathrm{mem}}^{\ell m} & =-\frac{G}{\sqrt{2} c^{l+2} R} U_{\mathrm{mem}}^{\ell m} \\
& =\frac{8 G m \nu}{c^{2} R} \times \sqrt{\frac{\pi}{5}} \mathrm{e}^{-\mathrm{i} m \psi} H_{\mathrm{mem}}^{\ell m} .
\end{aligned}
$$

With this convention, the memory pieces directly add to the waveform modes stated in Eq. (11). As the expressions are quite long, we present here only the $H_{\mathrm{DC}}^{20}$ mode to 3PN and leading order in eccentricity:

$$
\begin{aligned}
& H_{\mathrm{DC}}^{20}=-\frac{5}{14 \sqrt{6}}\left(H_{\mathrm{Newt}}^{20}+x H_{1 \mathrm{PN}}^{20}+x^{3 / 2} H_{1.5 \mathrm{PN}}^{20}+x^{2} H_{2 \mathrm{PN}}^{20}+x^{5 / 2} H_{2.5 \mathrm{PN}}^{20}+x^{3} H_{3 \mathrm{PN}}^{20}\right) \\
& H_{\text {Newt }}^{20}=1-\left(\frac{e}{e_{i}}\right)^{12 / 19} \\
& H_{1 \mathrm{PN}}^{20}=-\frac{4075}{4032}+\frac{67 \nu}{48}+\left(\frac{e}{e_{i}}\right)^{12 / 19}\left(-\frac{2833}{3192}+\frac{197 \nu}{114}\right)+\left(\frac{e}{e_{i}}\right)^{24 / 19}\left(\frac{145417}{76608}-\frac{2849 \nu}{912}\right) \\
& H_{1.5 \mathrm{PN}}^{20}=-\frac{377 \pi}{228}\left(\frac{e}{e_{i}}\right)^{12 / 19}+\frac{377 \pi}{228}\left(\frac{e}{e_{i}}\right)^{30 / 19} \\
& H_{2 \mathrm{PN}}^{20}=-\frac{151877213}{67060224}-\frac{123815 \nu}{44352}+\frac{205 \nu^{2}}{352}+\left(\frac{e}{e_{i}}\right)^{12 / 19}\left(\frac{358353209}{366799104}-\frac{738407 \nu}{727776}-\frac{20597 \nu^{2}}{17328}\right) \\
& +\left(\frac{e}{e_{i}}\right)^{24 / 19}\left(\frac{411966361}{122266368}-\frac{825950 \nu}{68229}+\frac{561253 \nu^{2}}{51984}\right) \\
& +\left(\frac{e}{e_{i}}\right)^{36 / 19}\left(-\frac{50392977379}{24208740864}+\frac{764295307 \nu}{48033216}-\frac{11654209 \nu^{2}}{1143648}\right) \\
& H_{2.5 \mathrm{PN}}^{20}=-\frac{253 \pi}{336}+\frac{253 \pi \nu}{84}+\left(\frac{e}{e_{i}}\right)^{12 / 19}\left(\frac{3763903 \pi}{7277760}+\frac{12788779 \pi \nu}{1819440}\right)+\left(\frac{e}{e_{i}}\right)^{24 / 19}\left(\frac{54822209 \pi}{8733312}-\frac{1074073 \pi \nu}{103968}\right) \\
& +\left(\frac{e}{e_{i}}\right)^{30 / 19}\left(\frac{5340205 \pi}{1455552}-\frac{371345 \pi \nu}{51984}\right)+\left(\frac{e}{e_{i}}\right)^{42 / 19}\left(-\frac{424020733 \pi}{43666560}+\frac{27049187 \pi \nu}{3638880}\right) \\
& H_{3 \mathrm{PN}}^{20}=-\frac{4397711103307}{532580106240}+\left(\frac{700464542023}{13948526592}-\frac{205 \pi^{2}}{96}\right) \nu+\frac{69527951 \nu^{2}}{166053888}+\frac{1321981 \nu^{3}}{5930496} \\
& +\left(\frac{e}{e_{i}}\right)^{12 / 19}\left[-\frac{4942027570449143}{96592876047360}-\frac{81025 \pi^{2}}{103968}+\frac{3317 \gamma_{E}}{399}+\left(-\frac{10309531979}{7466981760}+\frac{3977 \pi^{2}}{3648}\right) \nu\right. \\
& \left.+\frac{267351733 \nu^{2}}{82966464}+\frac{772583 \nu^{3}}{2222316}+\frac{12091 \ln 2}{5985}+\frac{78003 \ln 3}{5320}+\frac{3317 \ln x}{798}\right]+\frac{710645 \pi^{2}}{103968}\left(\frac{e}{e_{i}}\right)^{30 / 19} \\
& +\left(\frac{e}{e_{i}}\right)^{24 / 19}\left(-\frac{31102835980319}{14049872879616}+\frac{279737759653 \nu}{167260391424}+\frac{26730466283 \nu^{2}}{1991195136}-\frac{397176241 \nu^{3}}{23704704}\right)
\end{aligned}
$$




$$
\begin{aligned}
& +\left(\frac{e}{e_{i}}\right)^{36 / 19}\left(-\frac{142763304914707}{25758100279296}+\frac{48901891428821 \nu}{919932152832}-\frac{400181473249 \nu^{2}}{3650524416}+\frac{2295879173 \nu^{3}}{43458624}\right) \\
& +\left(\frac{e}{e_{i}}\right)^{48 / 19}\left[\frac{385621605844415513}{5740376633671680}-\frac{157405 \pi^{2}}{25992}-\frac{3317 \gamma_{E}}{399}+\left(-\frac{49590995147570629}{478364719472640}+\frac{1271 \pi^{2}}{1216}\right) \nu\right. \\
& \left.+\frac{3194536246463 \nu^{2}}{34514049024}-\frac{1672948713 \nu^{3}}{45653504}-\frac{12091 \ln 2}{5985}-\frac{78003 \ln 3}{5320}-\frac{3317 \ln x}{798}-\frac{6634}{2527} \ln \left(\frac{e}{e_{i}}\right)\right] .
\end{aligned}
$$

All nonzero DC memory modes are presented to leading order in eccentricity in Appendix D and to $\mathcal{O}\left(e^{6}\right)$ in the Supplemental Material [67].

An important check is to take the circular limit of our calculated memory modes and compare to the circular 3PN memory modes computed in Ref. [62]. To illustrate this fact, we take the circular limit of the 20-mode stated in Eqs. (27) by setting $e=0$ and find

$$
\begin{aligned}
H_{\mathrm{DC}}^{20}= & -\frac{5}{14 \sqrt{6}}\left\{1+x\left(-\frac{4075}{4032}+\frac{67 \nu}{48}\right)+x^{2}\left(-\frac{151877213}{67060224}-\frac{123815 \nu}{44352}+\frac{205 \nu^{2}}{352}\right)+x^{5 / 2}\left(-\frac{253 \pi}{336}+\frac{253 \pi \nu}{84}\right)\right. \\
& \left.+x^{3}\left[-\frac{4397711103307}{532580106240}+\left(\frac{700464542023}{13948526592}-\frac{205 \pi^{2}}{96}\right) \nu+\frac{69527951 \nu^{2}}{166053888}+\frac{1321981 \nu^{3}}{5930496}\right]\right\},
\end{aligned}
$$

in perfect agreement with Eq. (4.3a) of Ref. [62]. The higher DC modes up to $\ell=10$ in the circular limit are consistent with Eq. (4.3) of Ref. [62] as well. Moreover, we can check the leading eccentricity part at Newtonian order against Eq. (2.35) in Ref. [63]. They are found to be equal. Note that at Newtonian order the computation of the DC memory is in principle possible for arbitrary eccentricities [see Eq. (2.34) in Ref. [63]]; however, this becomes difficult at higher PN orders, especially when tail terms come into play.

\section{Oscillatory memory}

Before considering the oscillatory integrals, let us recall some properties of the nonlinear memory. As mentioned at the beginning of Sec. II B, the memory contribution to the radiative mass multipole is formally of $2.5 \mathrm{PN}$ order. But due to the hereditary nature, the nonoscillatory terms are raised by $2.5 \mathrm{PN}$ orders to appear already at the Newtonian level. From the oscillatory terms we cannot expect the same behavior, due to the fact that the oscillations in the remote past effectively cancel each other out. Thus, we expect that only the recent past will contribute.

Examining the remaining oscillatory integrals, we notice that they are of the following form:

$$
U_{\mathrm{osc}}^{\ell m} \sim \int_{-\infty}^{T_{R}} \mathrm{~d} t x^{p}(t) e^{q}(t) \mathrm{e}^{\mathrm{i}\left(s \lambda_{\xi}+r_{\xi}^{\xi}\right)}
$$

Note that we have $s=-m$. Here we provide a formula to evaluate these integrals, its derivation is presented in Appendix C. Using the fact that $\lambda_{\xi}=(1+k) \xi$ and $\xi=$ $n t$ to the required PN order as well as the notion that the integral is essentially given by the contributions at the current time, we find

$$
U_{\mathrm{osc}}^{\ell m} \sim-\frac{\mathrm{i}}{n(r+s(1+k))} x^{p} e^{q} \mathrm{e}^{\mathrm{i}\left(s \lambda_{\xi}+r \xi\right)},
$$

where the time dependence on $T_{R}$ is not written explicitly. Expanding the denominator, we have to distinguish between two different cases. The first applies if $r \neq-s$; we then find

$$
U_{\mathrm{osc}}^{\ell m} \sim-\frac{\mathrm{i}}{r+s} x^{p-3 / 2} e^{q} \mathrm{e}^{\mathrm{i}\left(s \lambda_{\xi}+r \xi\right)} .
$$

Since $p=5$ at Newtonian order and the leading terms in the waveform are of order $x$, these integrals lead to $2.5 \mathrm{PN}$ contributions to the waveform. As we have expected, these kinds of terms oscillating on the orbital time scale keep their formal PN order, and we call them the fast oscillatory memory.

On the other hand, for $r=-s$ we find

$$
\begin{aligned}
U_{\mathrm{osc}}^{\ell m} \sim & -\frac{\mathrm{i}}{3 s}\left[x^{p-5 / 2}+x^{p-3 / 2}\left(-\frac{3}{2}+\frac{7 \nu}{3}\right)\right] \\
& \times e^{q} \mathrm{e}^{\mathrm{i} s\left(\lambda_{\xi}-\xi\right)}+\mathcal{O}\left(e^{q+2}\right) .
\end{aligned}
$$

This corresponds to terms that oscillate solely on the periastron precession time scale, and we therefore call these terms the slow oscillatory memory. Because of the much slower oscillations, they are enhanced by 1PN order (corresponding to the PN order of precession) and enter the waveform at 1.5PN. Note also that in Eq. (32) eccentricity corrections of $\mathcal{O}\left(e^{q+2}\right)$ appear, whereas Eq. (31) would 
only be affected by eccentricity corrections starting at 3.5PN order.

We provide the oscillatory memory contributions to the spherical harmonic modes in the same form as for the DC memory, according to Eq. (26). Besides the DC memory contribution, the 20-mode also contains fast oscillatory memory at $2.5 \mathrm{PN}$ :

$H_{\text {osc }}^{20}=\mathrm{i} \frac{16 \sqrt{6}}{7} \nu e x^{5 / 2}\left(-\mathrm{e}^{-\mathrm{i} \xi}+\mathrm{e}^{\mathrm{i} \xi}-\frac{647}{576} e \mathrm{e}^{-2 \mathrm{i} \xi}+\frac{647}{576} e \mathrm{e}^{2 \mathrm{i} \xi}\right)$.

Note that while the DC memory is purely real and therefore only affects the plus polarization (with the usual conventions on the polarization triad), the oscillatory contributions influence both polarizations.

In the $m \neq 0$ modes, only the oscillatory memory is present. For the dominant 22-mode we find

$$
\begin{aligned}
H_{\text {osc }}^{22}= & \mathrm{i} e^{2} \nu \mathrm{e}^{2 \mathrm{i} \xi}\left[-\frac{13}{252} x^{3 / 2}+\left(\frac{697}{336}-\frac{865 \nu}{216}\right) x^{5 / 2}-\frac{29 \pi}{126} x^{3}\right] \\
& +21 \mathrm{i} x^{5 / 2} e \nu\left[\frac{19}{6} e+\frac{4}{3} \mathrm{e}^{-\mathrm{i} \xi}-4 \mathrm{e}^{\mathrm{i} \xi}+\frac{65}{24} e \mathrm{e}^{-2 \mathrm{i} \xi}\right] .
\end{aligned}
$$

Here the slow oscillatory part in the first and second lines is proportional to $\mathrm{e}^{2 \mathrm{i} \xi}$, as we factored out $\mathrm{e}^{-2 \mathrm{i} \psi}$ according to Eq. (26). Three different PN orders of slow oscillatory memory terms appear in this mode. The first one at $1.5 \mathrm{PN}$ arises from the leading-order memory contribution to the radiative mass multipole at $2.5 \mathrm{PN}$, so as expected it is enhanced by one post-Newtonian order. At $2.5 \mathrm{PN}$, there is the 1PN correction to the first term as well as a part coming from the 1PN correction to the multipole. Finally, at 3PN there is a term originating from the $1.5 \mathrm{PN}$ correction to the memory part of the multipole; this corresponds to the memory of the gravitational-wave tail. The terms in the second line correspond to fast oscillatory memory entering at the $2.5 \mathrm{PN}$ level.

\section{FULL 3PN ECCENTRIC WAVEFORM}

In this section we summarize the results necessary to construct the full waveform for eccentric binaries at third post-Newtonian order, including all instantaneous, hereditary, and post-adiabatic contributions, as described in Sec. V of Paper I. Rather than listing the lengthy expressions, we give an overview at which PN order the individual terms enter the waveform and where they can be found. Explicit expressions for all spherical harmonic modes are given in a supplemental Mathematica notebook [67].
We present the waveform in terms of the secular evolving PN parameter $\bar{x}$ and the time eccentricity $\bar{e}$, parametrized by the angles $\xi$ and $\psi$. We refer to Sec. V C of Paper I for their definition, and to Appendix B therein for various relations between the orbital elements $(l, \lambda, \phi)$ and $\left(\xi, \lambda_{\xi}, \psi\right)$. The secular evolution of the parameters $\bar{x}$ and $\bar{e}$ is given in Appendix B. The spherical harmonic modes describing the waveform are then written in the following form:

$$
h^{\ell m}=\frac{8 G m \nu}{c^{2} R} \bar{x} \sqrt{\frac{\pi}{5}} \mathrm{e}^{-\mathrm{i} m \psi} H^{\ell m} .
$$

Modes with $m<0$ can be calculated from

$$
h^{\ell-m}=(-1)^{\ell} \bar{h}^{\ell m} .
$$

In general, the individual modes can be split into three types of contributions:

$$
H^{\ell m}=H_{\text {inst }}^{\ell m}+H_{\text {hered }}^{\ell m}+H_{\text {post-ad }}^{\ell m} .
$$

The instantaneous terms depend only on the instantaneous state of the source at a given retarded time, with contributions at different orders relative to the leading order for each mode given as

$$
\begin{aligned}
H_{\text {inst }}^{\ell m}= & \left(H_{\text {inst }}^{\ell m}\right)_{\text {Lead }}+\left(H_{\text {inst }}^{\ell m}\right)_{1 \mathrm{PN}}+\left(H_{\text {inst }}^{\ell m}\right)_{1.5 \mathrm{PN}} \\
& +\left(H_{\text {inst }}^{\ell m}\right)_{2 \mathrm{PN}}+\left(H_{\text {inst }}^{\ell m}\right)_{2.5 \mathrm{PN}}+\left(H_{\text {inst }}^{\ell m}\right)_{3 \mathrm{PN}} .
\end{aligned}
$$

These are given in terms of $x, e$, and $u$ in Eqs. (5.09)-(5.11) and Eq. (A1) of Ref. [65]. The parametrization in terms of $u$ has to be transformed to $\xi$ using Eq. (B2b) in Paper I.

The post-adiabatic contributions are introduced by radiation-reaction corrections to the quasi-Keplerian parametrization, at relative $2.5 \mathrm{PN}$ order:

$$
H_{\text {post-ad }}^{\ell m}=\left(H_{\text {post-ad }}^{\ell m}\right)_{2.5 \mathrm{PN}} \text {. }
$$

They are given in Eqs. (66)-(67) of Paper I.

The hereditary contributions, on the other hand, depend on the entire dynamical past of the binary system. They can be further split into tail and memory parts:

$$
H_{\text {hered }}^{\ell m}=H_{\text {tail }}^{\ell m}+H_{\text {mem }}^{\ell m} \text {. }
$$

For the tails we find contributions at different orders relative to the leading order for each mode as

$$
H_{\text {tail }}^{\ell m}=\left(H_{\text {tail }}^{\ell m}\right)_{1.5 \mathrm{PN}}+\left(H_{\text {tail }}^{\ell m}\right)_{2.5 \mathrm{PN}}+\left(H_{\text {tail }}^{\ell m}\right)_{3 \mathrm{PN}} .
$$

These are given in Eqs. (47)-(48) of Paper I.

There is both DC memory and oscillatory memory:

$$
H_{\mathrm{mem}}^{\ell m}=H_{\mathrm{DC}}^{\ell 0}+H_{\mathrm{osc}}^{\ell m} .
$$


DC memory enters the waveform in the $m=0$ modes at all relative orders,

$$
\begin{aligned}
H_{\mathrm{DC}}^{\ell 0}= & \left(H_{\mathrm{DC}}^{\ell 0}\right)_{\mathrm{Lead}}+\left(H_{\mathrm{DC}}^{\ell 0}\right)_{1 \mathrm{PN}}+\left(H_{\mathrm{DC}}^{\ell 0}\right)_{1.5 \mathrm{PN}} \\
& +\left(H_{\mathrm{DC}}^{\ell 0}\right)_{2 \mathrm{PN}}+\left(H_{\mathrm{DC}}^{\ell 0}\right)_{2.5 \mathrm{PN}}+\left(H_{\mathrm{DC}}^{\ell 0}\right)_{3 \mathrm{PN}},
\end{aligned}
$$

while slow and fast oscillatory memory enter as

$$
\begin{aligned}
H_{\mathrm{osc}}^{\ell m}= & \left(H_{\text {slow osc }}^{\ell m}\right)_{1.5 \mathrm{PN}}+\left(H_{\text {slow osc }}^{\ell m}\right)_{2.5 \mathrm{PN}} \\
& +\left(H_{\text {slow osc }}^{\ell m}\right)_{3 \mathrm{PN}}+\left(H_{\text {fast osc }}^{\ell m}\right)_{2.5 \mathrm{PN}}
\end{aligned}
$$

Slow oscillatory memory is due to the double-periodic nature of eccentric motion and is not present in quasicircular binary systems. All memory modes are computed in this paper and are listed in Appendixes D and E.

As an example, we present here the dominant $H^{22}$ mode including all contributions to $\mathcal{O}(e)$ :

$$
\begin{aligned}
H_{\text {Newt }}^{22}= & +\bar{e}\left(\frac{1}{4} \mathrm{e}^{-\mathrm{i} \xi}+\frac{5}{4} \mathrm{e}^{\mathrm{i} \xi}\right) \\
H_{1 \mathrm{PN}}^{22}= & \bar{x}\left\{-\frac{107}{42}+\frac{55 \nu}{42}+\bar{e}\left[\mathrm{e}^{-\mathrm{i} \xi}\left(-\frac{257}{168}+\frac{169 \nu}{168}\right)+\mathrm{e}^{\mathrm{i} \xi}\left(-\frac{31}{24}+\frac{35 \nu}{24}\right)\right]\right\} \\
H_{1.5 \mathrm{PN}}^{22}= & \bar{x}^{3 / 2}\left\{2 \pi+\bar{e}\left[\mathrm{e}^{-\mathrm{i} \xi}\left(\frac{11 \pi}{4}+\frac{27 \mathrm{i}}{2} \ln \left(\frac{3}{2}\right)\right)+\mathrm{e}^{\mathrm{i} \xi}\left(\frac{13 \pi}{4}+\frac{3 \mathrm{i}}{2} \ln (2)\right)\right]\right\} \\
H_{2 \mathrm{PN}}^{22}= & \bar{x}^{2}\left\{-\frac{2173}{1512}-\frac{1069 \nu}{216}+\frac{2047 \nu^{2}}{1512}+\bar{e}\left[\mathrm{e}^{\mathrm{i} \xi}\left(-\frac{2155}{252}-\frac{1655 \nu}{672}+\frac{371 \nu^{2}}{288}\right)+\mathrm{e}^{-\mathrm{i} \xi}\left(-\frac{4271}{756}-\frac{35131 \nu}{6048}+\frac{421 \nu^{2}}{864}\right)\right]\right\} \\
H_{2.5 \mathrm{PN}}^{22}= & \bar{x}^{5 / 2}\left\{-\frac{107 \pi}{21}+\left(-24 \mathrm{i}+\frac{34 \pi}{21}\right) \nu+\bar{e}\left[\mathrm{e}^{\mathrm{i} \xi}\left(-\frac{9 \mathrm{i}}{2}+\frac{229 \pi}{168}+\left(-\frac{14579 \mathrm{i}}{140}+\frac{61 \pi}{42}\right) \nu+\left(\frac{473 \mathrm{i}}{28}-\frac{3 \mathrm{i} \nu}{7}\right) \ln (2)\right)\right.\right. \\
& \left.\left.+\mathrm{e}^{-\mathrm{i} \xi}\left(-\frac{27 \mathrm{i}}{2}-\frac{1081 \pi}{168}+\left(-\frac{1291 \mathrm{i}}{180}+\frac{137 \pi}{42}\right) \nu+\left(\frac{27 \mathrm{i}}{4}+9 \mathrm{i} \nu\right) \ln \left(\frac{3}{2}\right)\right)\right]\right\} \\
& \left.\left.\left.+\left(-\frac{6527}{210}+\frac{3 \mathrm{i} \pi}{2}\right) \ln (2)+\frac{3 \ln 2(2)}{2}-\frac{3103 \ln (\bar{x})}{420}\right)\right]\right\} \cdot \\
H_{3 \mathrm{PN}}^{22}= & \bar{x}^{3}\left\{\frac{27027409}{646800}+\frac{428 \mathrm{i} \pi}{105}+\frac{2 \pi^{2}}{3}-\frac{856 \gamma_{E}}{105}+\left(-\frac{278185}{33264}+\frac{41 \pi^{2}}{96}\right) \nu-\frac{20261 \nu^{2}}{2772}+\frac{114635 \nu^{3}}{99792}-\frac{1712 \ln (2)}{105}-\frac{428 \ln (\bar{x})}{105}\right. \\
+ & \bar{e}\left[\mathrm { e } ^ { - \mathrm { i } \xi } \left(\frac{219775769}{1663200}+\frac{749 \mathrm{i} \pi}{60}+\frac{49 \pi^{2}}{24}-\frac{749 \gamma_{E}}{30}+\left(-\frac{121717}{20790}-\frac{41 \pi^{2}}{192}\right) \nu-\frac{86531 \nu^{2}}{8316}-\frac{33331 \nu^{3}}{399168}\right.\right. \\
+ & \left.\left(-\frac{2889}{70}+\frac{81 \mathrm{i} \pi}{2}\right) \ln \left(\frac{3}{2}\right)-\frac{81}{2} \ln { }^{2}\left(\frac{3}{2}\right)-\frac{749 \ln (2)}{15}-\frac{749 \ln (\bar{x})}{60}\right) \\
+ & \mathrm{e}^{\mathrm{i} \xi}\left(\frac{55608313}{1058400}+\frac{3103 \mathrm{i} \pi}{420}+\frac{29 \pi^{2}}{24}-\frac{3103 \gamma_{E}}{210}+\left(-\frac{199855}{3024}+\frac{41 \pi^{2}}{48}\right) \nu-\frac{9967 \nu^{2}}{1008}+\frac{35579 \nu^{3}}{36288}\right. \\
& (45 \mathrm{f}) \\
& (45 \mathrm{e}) \\
&
\end{aligned}
$$

Note here the difference at 2.5PN order between Eq. (40) and Eq. (76) of Paper I, due to additional memory terms not yet considered in Paper I. Complete expressions for all modes to $\mathcal{O}\left(e^{6}\right)$ are given in the Supplemental Material [67].

By taking the quasicircular limit of our modes as described in Sec. V E of Paper I, we can compare the instantaneous, tail, and (fast) oscillatory memory contributions of our waveform modes with Ref. [64] and the DC memory terms with Ref. [62]. In all of them we find perfect agreement.

\section{BRIEF SUMMARY}

In this paper we computed the memory contribution to the gravitational waveform from nonspinning compact binaries in eccentric orbits at the third post-Newtonian order. Our results complete the previous work on the instantaneous parts [65] 
and on the tail and post-adiabatic contributions [66]. These waveforms form the basis for the construction of increasingly accurate GW templates from binary systems in eccentric orbits.

There are two fundamentally different types of memory. DC memory is a slowly increasing, nonoscillatory contribution to the gravitational-wave amplitude, entering at Newtonian order, leading to a difference in the amplitude between early and late times. Oscillatory memory, on the other hand, enters at higher PN orders as a normal periodic contribution. Due to the double-periodic nature of the eccentric motion, slow oscillatory memory contributions on the periastron precession time scale are enhanced by a factor of $1 \mathrm{PN}$, and thus already enter the waveform at 1.5PN order. This is unlike the quasicircular case, where oscillatory memory only enters at $2.5 \mathrm{PN}$ order.

\section{ACKNOWLEDGMENTS}

We thank Marc Favata for an early review and useful comments. We also thank Maria Haney and Achamveedu Gopakumar for insightful discussions and comments, as well as Luc Blanchet for stimulating discussions. M. E. and Y.B. are supported by the Swiss National Science Foundation. Y. B. is supported by a Forschungskredit of the University of Zurich, Grant No. FK-18-084.

\section{APPENDIX A: COMPUTATION OF THE MEMORY VIA THE RADIATIVE MASS MULTIPOLES}

The computation of the nonlinear memory in the paper is done effectively via the GW energy flux with the formula given in Eq. (8). An alternative way is to directly compute the required moments of the memory contribution to the radiative mass multipole. The leading-order memory piece of the mass quadrupole moment contributes at $2.5 \mathrm{PN}$; however, due to the hereditary integral the DC terms are raised by $2.5 \mathrm{PN}$ orders such that they contribute at leading order in the waveform polarization. Reference [81] lists the memory contributions up to $3.5 \mathrm{PN}$. From this we are able to compute the DC memory to $1 \mathrm{PN}$ accuracy. The hereditary integral enhances the slow oscillatory memory terms by $1 \mathrm{PN}$; therefore, by knowing the $3.5 \mathrm{PN}$ contribution to the mass moments we find the leading-order 2.5PN terms contributing at $1.5 \mathrm{PN}$ and $2.5 \mathrm{PN}$ in the waveform, and that the 3PN terms appear at 2PN and 3PN and the $3.5 \mathrm{PN}$ terms at $2.5 \mathrm{PN}$. However, what we miss are the $4 \mathrm{PN}$ terms that appear in the waveform at $3 \mathrm{PN}$ level. On the other hand, the fast oscillatory memory is not affected by the hereditary integral in its PN order, and we recover it at $2.5 \mathrm{PN}$ and 3PN. The required memory contributions at $3.5 \mathrm{PN}$ to the radiative mass moments are

$$
\begin{aligned}
U_{i j}^{\text {mem }}\left(T_{R}\right)= & \frac{G}{c^{5}} \int_{-\infty}^{T_{R}} \mathrm{~d} \tau\left[-\frac{2}{7} M_{a\langle i}^{(3)}(\tau) M_{j\rangle a}^{(3)}(\tau)\right]+\frac{G}{c^{7}} \int_{-\infty}^{T_{R}} \mathrm{~d} \tau\left[-\frac{5}{756} M_{a b}^{(4)}(\tau) M_{i j a b}^{(4)}(\tau)-\frac{32}{63} S_{a\langle i}^{(3)}(\tau) S_{j\rangle a}^{(3)}(\tau)\right. \\
& \left.+\varepsilon_{a b\langle i}\left(\frac{5}{42} S_{j\rangle b c}^{(4)}(\tau) M_{a c}^{(3)}(\tau)-\frac{20}{189} M_{j\rangle b c}^{(4)}(\tau) S_{a c}^{(3)}(\tau)\right)\right] \\
U_{i j k}^{\operatorname{mem}}\left(T_{R}\right)= & \frac{G}{c^{5}} \int_{-\infty}^{T_{R}} \mathrm{~d} \tau\left[-\frac{1}{3} M_{a\langle i}^{(3)}(\tau) M_{j k\rangle a}^{(4)}(\tau)-\frac{4}{5} \varepsilon_{a b\langle i} M_{j a}^{(3)}(\tau) S_{k\rangle b}^{(3)}(\tau)\right] \\
U_{i j k l}^{\operatorname{mem}}\left(T_{R}\right)= & \frac{G}{c^{3}} \int_{-\infty}^{T_{R}} \mathrm{~d} \tau\left[\frac{2}{5} M_{\langle i j}^{(3)}(\tau) M_{k l\rangle}^{(3)}(\tau)\right]+\frac{G}{c^{5}} \int_{-\infty}^{T_{R}} \mathrm{~d} \tau\left[\frac{12}{55} M_{a\langle i}^{(4)}(\tau) M_{j k l\rangle a}^{(4)}(\tau)-\frac{14}{99} M_{a\langle i j}^{(4)}(\tau) M_{k l\rangle a}^{(4)}(\tau)\right. \\
& \left.+\frac{32}{45} S_{\langle i j}^{(3)}(\tau) S_{k l\rangle}^{(3)}(\tau)+\varepsilon_{a b\langle i}\left(-\frac{4}{5} M_{j a}^{(3)}(\tau) S_{k l\rangle b}^{(4)}(\tau)+\frac{32}{45} S_{j a}^{(3)}(\tau) M_{k l\rangle b}^{(4)}(\tau)\right)\right] \\
U_{i j k l m}^{\text {mem }}\left(T_{R}\right)= & \frac{G}{c^{3}} \int_{-\infty}^{T_{R}} \mathrm{~d} \tau\left[\frac{20}{21} M_{\langle i j}^{(3)}(\tau) M_{k l m\rangle}^{(4)}(\tau)\right], \\
U_{i j k l m n}^{\operatorname{mem}}\left(T_{R}\right)= & \frac{G}{c^{3}} \int_{-\infty}^{T_{R}} \mathrm{~d} \tau\left[\frac{5}{7} M_{\langle i j k}^{(4)}(\tau) M_{l m n\rangle}^{(4)}(\tau)-\frac{15}{14} M_{\langle i j}^{(3)}(\tau) M_{k l m n\rangle}^{(4)}(\tau)\right] .
\end{aligned}
$$

Note that the symmetric trace-free (STF) projection $\langle\ldots\rangle$ only applies to the free indices $i j k \ldots$. The integrand in those equations consists of products of canonical mass and current moments, $M_{L}^{(n)}(\tau)$ and $S_{L}^{(n)}(\tau)$, and the superscript in brackets stands for the $n$th derivative with respect to $\tau$. The canonical moments are related by a gauge transformation to the source moments $I_{L}$ and $J_{L}$ along with some more gauge moments that enter at $2.5 \mathrm{PN}$ in the $\delta I_{L}, \delta J_{L}$ terms,

$$
M_{L}=I_{L}+G \delta I_{L}+\mathcal{O}\left(G^{2}\right),
$$




$$
S_{L}=J_{L}+G \delta J_{L}+\mathcal{O}\left(G^{2}\right) .
$$

For our purpose of calculating the memory contribution to next-to-leading order, we only need the 1PN part of the source moments. Here we list the relevant source moments at $1 \mathrm{PN}$ for two nonspinning compact objects in general orbits [65]. The source moments are written in terms of $x_{i}$ and $v_{i}$, which denote the binary's relative separation and relative velocity. Moreover, $r$ is the distance between the two objects, and thus $r=|\boldsymbol{x}|$ and $\dot{r}$ is the radial velocity. For the mass quadrupole moment we have

$$
I_{i j}=\nu m\left[A_{1} x_{\langle i} x_{j\rangle}+A_{2} \frac{r \dot{r}}{c^{2}} x_{\langle i} x_{j\rangle}+A_{3} \frac{r^{2}}{c^{2}} v_{\langle i} v_{j\rangle}\right],
$$

where

$$
\begin{aligned}
& A_{1}=1+\frac{1}{c^{2}}\left[v^{2}\left(\frac{29}{42}-\frac{29 \nu}{14}\right)+\frac{G m}{r}\left(-\frac{5}{7}+\frac{8 \nu}{7}\right)\right], \\
& A_{2}=-\frac{4}{7}+\frac{12 \nu}{7} \\
& A_{3}=\frac{11}{21}-\frac{11 \nu}{7} .
\end{aligned}
$$

The 1PN mass octupole is

$I_{i j k}=-\nu m \Delta\left[B_{1} x_{\langle i j k\rangle}+B_{2} \frac{r \dot{r}}{c^{2}} x_{\langle i j} v_{k\rangle}+B_{3} \frac{r^{2}}{c^{2}} x_{\langle i} v_{j k\rangle}\right]$,

where

$$
\begin{aligned}
& B_{1}=1+\frac{1}{c^{2}}\left[v^{2}\left(\frac{5}{6}-\frac{19 \nu}{6}\right)+\frac{G m}{r}\left(-\frac{5}{6}+\frac{13 \nu}{6}\right)\right], \\
& B_{2}=-(1-2 \nu), \\
& B_{3}=1-2 \nu,
\end{aligned}
$$

and $\Delta=\left(m_{1}-m_{2}\right) / m$ is the mass difference ratio. Moreover, we need also the leading-Newtonian-order part of the mass hexadecapole,

$$
I_{i j k l}=\nu m x_{\langle i j k l\rangle}(1-3 \nu) \text {. }
$$

From the current source moments we need the quadrupole, which is

$$
J_{i j}=-\nu m \Delta\left[C_{1} \varepsilon_{a b\langle i} x_{j\rangle a} v_{b}+C_{2} \frac{r \dot{r}}{c^{2}} \varepsilon_{a b\langle i} v_{j\rangle b} x_{a}\right],
$$

where

$$
\begin{gathered}
C_{1}=1+\frac{1}{c^{2}}\left[v^{2}\left(\frac{13}{28}-\frac{17 \nu}{7}\right)+\frac{G m}{r}\left(\frac{27}{14}+\frac{15 \nu}{7}\right)\right], \\
C_{2}=\frac{5}{28}(1-2 \nu),
\end{gathered}
$$

and finally the leading order of the current octupole is

$$
J_{i j k}=\nu m \varepsilon_{a b\langle i} x_{j k\rangle a} v_{b}(1-3 \nu) .
$$

Having the source moments in hand (and thus in our case also the canonical moments), we can calculate the products of time derivatives of the canonical moments occurring in the integrands of Eq. (A1a)-(A1e). Before treating the hereditary integral, we transform from the STF moments $U_{L}^{\text {mem }}$ computed here to the scalar version of the radiative mass moments using Eq. (4) of Paper I. These are the same moments that we find when computing the memory with Eq. (8). The hereditary integral is evaluated in the same way as described in Secs. III B and III C. Using this method, we find the 1PN DC memory and the 1PN oscillatory memory. Be aware that the DC memory appears in the waveform at leading Newtonian order, while the first slow oscillatory memory terms appear at $1.5 \mathrm{PN}$ and the fast oscillatory memory at $2.5 \mathrm{PN}$.

This method of computing the memory contribution serves as a check. We can compare the relative 1PN pieces of the DC and oscillatory memory calculated before and here, and they are found to be in perfect agreement.

\section{APPENDIX B: RADIATION-REACTION EVOLUTION EQUATIONS}

In this Appendix we provide the secular 3PN-accurate evolution equations for $x$ and $e$ [78-80] in MH gauge. The instantaneous terms are exact, whereas the eccentricity enhancement functions appearing in the hereditary contributions are given in an eccentricity expansion. We begin by listing the pieces needed for the evolution of $x$ :

$$
\frac{\mathrm{d} x}{\mathrm{~d} t}=\frac{2 c^{3} \nu x^{5}}{3 G m}\left(\mathcal{X}_{\text {Newt }}+x \mathcal{X}_{1 \mathrm{PN}}+x^{2} \mathcal{X}_{2 \mathrm{PN}}+x^{3} \mathcal{X}_{3 \mathrm{PN}}+\mathcal{X}_{\text {hered }}\right),
$$

where

$$
\begin{aligned}
\mathcal{X}_{\text {Newt }}= & \frac{1}{\left(1-e^{2}\right)^{7 / 2}}\left\{\frac{96}{5}+\frac{292 e^{2}}{5}+\frac{37 e^{4}}{5}\right\}, \\
\mathcal{X}_{1 \mathrm{PN}}= & \frac{1}{\left(1-e^{2}\right)^{9 / 2}}\left\{-\frac{1486}{35}-\frac{264 \nu}{5}+e^{2}\left(\frac{2193}{7}-570 \nu\right)\right. \\
& \left.+e^{4}\left(\frac{12217}{20}-\frac{5061 \nu}{10}\right)+e^{6}\left(\frac{11717}{280}-\frac{148 \nu}{5}\right)\right\},
\end{aligned}
$$




$$
\begin{aligned}
\mathcal{X}_{2 \mathrm{PN}}= & \frac{1}{\left(1-e^{2}\right)^{11 / 2}}\left\{-\frac{11257}{945}+\frac{15677 \nu}{105}+\frac{944 \nu^{2}}{15}+e^{2}\left(-\frac{2960801}{945}-\frac{2781 \nu}{5}+\frac{182387 \nu^{2}}{90}\right)\right. \\
& +e^{4}\left(-\frac{68647}{1260}-\frac{1150631 \nu}{140}+\frac{396443 \nu^{2}}{72}\right)+e^{6}\left(\frac{925073}{336}-\frac{199939 \nu}{48}+\frac{192943 \nu^{2}}{90}\right) \\
& +e^{8}\left(\frac{391457}{3360}-\frac{6037 \nu}{56}+\frac{2923 \nu^{2}}{45}\right)+\sqrt{1-e^{2}}\left[48-\frac{96 \nu}{5}+e^{2}\left(2134-\frac{4268 \nu}{5}\right)\right. \\
& \left.\left.+e^{4}\left(2193-\frac{4386 \nu}{5}\right)+e^{6}\left(\frac{175}{2}-35 \nu\right)\right]\right\}
\end{aligned}
$$

$$
\begin{aligned}
& \mathcal{X}_{3 \mathrm{PN}}=\frac{1}{\left(1-e^{2}\right)^{13 / 2}}\left\{\frac{614389219}{148500}+\left(-\frac{57265081}{11340}+\frac{369 \pi^{2}}{2}\right) \nu-\frac{16073 \nu^{2}}{140}-\frac{1121 \nu^{3}}{27}\right. \\
& +e^{2}\left(\frac{19769277811}{693000}+\left(\frac{66358561}{3240}+\frac{42571 \pi^{2}}{80}\right) \nu-\frac{3161701 \nu^{2}}{840}-\frac{1287385 \nu^{3}}{324}\right) \\
& +e^{4}\left(-\frac{3983966927}{8316000}+\left(\frac{6451690597}{90720}-\frac{12403 \pi^{2}}{64}\right) \nu+\frac{34877019 \nu^{2}}{1120}-\frac{33769597 \nu^{3}}{1296}\right) \\
& +e^{6}\left(-\frac{4548320963}{5544000}+\left(-\frac{59823689}{4032}-\frac{242563 \pi^{2}}{640}\right) \nu+\frac{411401857 \nu^{2}}{6720}-\frac{3200965 \nu^{3}}{108}\right) \\
& +e^{8}\left(\frac{19593451667}{2464000}+\left(-\frac{6614711}{480}-\frac{12177 \pi^{2}}{640}\right) \nu+\frac{92762 \nu^{2}}{7}-\frac{982645 \nu^{3}}{162}\right) \\
& +e^{10}\left(\frac{33332681}{197120}-\frac{1874543 \nu}{10080}+\frac{109733 \nu^{2}}{840}-\frac{8288 \nu^{3}}{81}\right)+\sqrt{1-e^{2}}\left[\left(-\frac{1425319}{1125}+\left(\frac{9874}{105}-\frac{41 \pi^{2}}{10}\right) \nu+\frac{632 \nu^{2}}{5}\right)\right. \\
& +e^{2}\left(\frac{933454}{375}+\left(-\frac{2257181}{63}+\frac{45961 \pi^{2}}{240}\right) \nu+\frac{125278 \nu^{2}}{15}\right) \\
& +e^{4}\left(\frac{840635951}{21000}+\left(-\frac{4927789}{60}+\frac{6191 \pi^{2}}{32}\right) \nu+\frac{317273 \nu^{2}}{15}\right) \\
& \left.+e^{6}\left(\frac{702667207}{31500}+\left(-\frac{6830419}{252}+\frac{287 \pi^{2}}{960}\right) \nu+\frac{232177 \nu^{2}}{30}\right)+e^{8}\left(\frac{56403}{112}-\frac{427733 \nu}{840}+\frac{4739 \nu^{2}}{30}\right)\right] \\
& \left.+\log \left[\frac{x\left(1+\sqrt{1-e^{2}}\right)}{x_{0}\left(2\left(1-e^{2}\right)\right)}\right]\left(\frac{54784}{175}+\frac{465664 e^{2}}{105}+\frac{4426376 e^{4}}{525}+\frac{1498856 e^{6}}{525}+\frac{31779 e^{8}}{350}\right)\right\}
\end{aligned}
$$$$
\mathcal{X}_{\text {hered }}=\frac{96}{5}\left\{4 \pi x^{3 / 2} \varphi(e)+\pi x^{5 / 2}\left[-\frac{4159}{672} \psi_{\omega}(e)-\frac{189}{8} \nu \zeta_{\omega}(e)\right]\right.
$$$$
\left.+x^{3}\left[-\frac{116761}{3675} \kappa(e)+\left(\frac{16 \pi^{2}}{3}-\frac{1712 \gamma_{E}}{105}-\frac{1712}{105} \log \left(\frac{4 x^{3 / 2}}{x_{0}}\right)\right) F(e)\right]\right\} \text {. }
$$

The helper functions appearing in the hereditary contribution are given by

$$
\begin{gathered}
\psi_{\omega}(e)=\frac{1344}{4159} \frac{1}{\left(1-e^{2}\right)^{3 / 2}}\left[\sqrt{1-e^{2}}\left(1-5 e^{2}\right) \varphi(e)-4 \tilde{\varphi}(e)\right]+\frac{8191}{4159} \psi(e), \\
\zeta_{\omega}(e)=\frac{583}{567} \zeta(e)-\frac{16}{567} \varphi(e) .
\end{gathered}
$$

The various enhancement functions appearing in these equations are listed below.

Next we state the evolution equation for the eccentricity. Note that we observed errors in the 2PN- and 3PN-order expressions in Eqs. (C10) and (C11) of Ref. [80]. These are likely due to the fact that only the relation between $e^{\mathrm{MH}}$ and $e^{\mathrm{ADM}}$ was inserted, but one also has to transform $\mathrm{d} e^{\mathrm{ADM}} / \mathrm{d} t$ to $\mathrm{d} e^{\mathrm{MH}} / \mathrm{d} t$, 


$$
\frac{\mathrm{d} e}{\mathrm{~d} t}=-\frac{c^{3} \nu e x^{4}}{G m}\left(\mathcal{E}_{\mathrm{N}}+x \mathcal{E}_{1 \mathrm{PN}}+x^{2} \mathcal{E}_{2 \mathrm{PN}}+x^{3} \mathcal{E}_{3 \mathrm{PN}}+\mathcal{E}_{\text {hered }}\right)
$$

where

$$
\begin{aligned}
& \mathcal{E}_{\text {Newt }}=\frac{1}{\left(1-e^{2}\right)^{5 / 2}}\left\{\frac{304}{15}+\frac{121 e^{2}}{15}\right\} \\
& \mathcal{E}_{1 \mathrm{PN}}=\frac{1}{\left(1-e^{2}\right)^{7 / 2}}\left\{-\frac{939}{35}-\frac{4084 \nu}{45}+e^{2}\left(\frac{29917}{105}-\frac{7753}{30} \nu\right)+e^{4}\left(\frac{13929}{280}-\frac{1664 \nu}{45}\right)\right\}, \\
& \mathcal{E}_{2 \mathrm{PN}}=\frac{1}{\left(1-e^{2}\right)^{9 / 2}}\left\{-\frac{949877}{1890}+\frac{18763 \nu}{42}+\frac{752 \nu^{2}}{5}+e^{2}\left(-\frac{3082783}{2520}-\frac{988423 \nu}{840}+\frac{64433 \nu^{2}}{40}\right)\right. \\
& +e^{4}\left(\frac{23289859}{15120}-\frac{13018711 \nu}{5040}+\frac{127411 \nu^{2}}{90}\right)+e^{6}\left(\frac{420727}{3360}-\frac{362071 \nu}{2520}+\frac{821 \nu^{2}}{9}\right) \\
& \left.+\sqrt{1-e^{2}}\left[\frac{1336}{3}-\frac{2672 \nu}{15}+e^{2}\left(\frac{2321}{2}-\frac{2321 \nu}{5}\right)+e^{4}\left(\frac{565}{6}-\frac{113 \nu}{3}\right)\right]\right\} \\
& \mathcal{E}_{3 \mathrm{PN}}=\frac{1}{\left(1-e^{2}\right)^{11 / 2}}\left\{\frac{54208557619}{6237000}+\left(\frac{50099023}{113400}+\frac{779 \pi^{2}}{10}\right) \nu-\frac{4088921 \nu^{2}}{2520}-\frac{61001 \nu^{3}}{486}\right. \\
& +e^{2}\left(\frac{46226320013}{6237000}+\left(\frac{28141879}{900}-\frac{139031 \pi^{2}}{960}\right) \nu-\frac{21283907 \nu^{2}}{3024}-\frac{86910509 \nu^{3}}{19440}\right) \\
& +e^{4}\left(-\frac{116987170177}{16632000}+\left(\frac{11499615139}{907200}-\frac{271871 \pi^{2}}{1920}\right) \nu+\frac{61093675 \nu^{2}}{4032}-\frac{2223241 \nu^{3}}{180}\right) \\
& +e^{6}\left(\frac{5891934893}{1232000}+\left(-\frac{5028323}{560}-\frac{6519 \pi^{2}}{640}\right) \nu+\frac{24757667 \nu^{2}}{2520}-\frac{11792069 \nu^{3}}{2430}\right) \\
& +e^{8}\left(\frac{302322169}{1774080}-\frac{1921387 \nu}{10080}+\frac{41179 \nu^{2}}{216}-\frac{193396 \nu^{3}}{1215}\right) \\
& +\sqrt{1-e^{2}}\left[-\frac{22713049}{15750}+\left(-\frac{5526991}{945}+\frac{8323 \pi^{2}}{180}\right) \nu+\frac{54332 \nu^{2}}{45}\right. \\
& +e^{2}\left(\frac{89395687}{7875}+\left(-\frac{38295557}{1260}+\frac{94177 \pi^{2}}{960}\right) \nu+\frac{681989 \nu^{2}}{90}\right) \\
& +e^{4}\left(\frac{5321445613}{378000}+\left(-\frac{26478311}{1512}+\frac{2501 \pi^{2}}{2880}\right) \nu+\frac{225106 \nu^{2}}{45}\right) \\
& \left.+e^{6}\left(\frac{186961}{336}-\frac{289691 \nu}{504}+\frac{3197 \nu^{2}}{18}\right)\right]+\frac{730168}{23625\left(1+\sqrt{1-e^{2}}\right)} \\
& \left.+\frac{304}{15}\left(\frac{82283}{1995}+\frac{297674}{1995} e^{2}+\frac{1147147}{15960} e^{4}+\frac{61311}{21280} e^{6}\right) \ln \left[\frac{x\left(1+\sqrt{1-e^{2}}\right)}{2 x_{0}\left(1-e^{2}\right)}\right]\right\}, \\
& \mathcal{E}_{\text {hered }}=-\frac{32}{5}\left\{-\frac{985}{48} \pi x^{3 / 2} \varphi_{e}(e)+\pi x^{5 / 2}\left[\frac{55691}{1344} \psi_{e}(e)+\frac{19067}{126} \nu \zeta_{e}(e)\right]\right. \\
& +x^{3}\left[\left(\frac{89789209}{352800}-\frac{87419 \ln 2}{630}+\frac{78003 \ln 3}{560}\right) \kappa_{e}(e)\right. \\
& \left.\left.-\frac{769}{96}\left(\frac{16 \pi^{2}}{3}-\frac{1712 \gamma_{E}}{105}-\frac{1712}{105} \ln \left(\frac{4 x^{3 / 2}}{x_{0}}\right)\right) F_{e}(e)\right]\right\} \text {. }
\end{aligned}
$$


The additional functions in the hereditary contribution are

$$
\begin{aligned}
& \varphi_{e}(e)=\frac{192}{985} \frac{\sqrt{1-e^{2}}}{e^{2}}\left[\sqrt{1-e^{2}} \varphi(e)-\tilde{\varphi}(e)\right], \\
& \psi_{e}(e)=\frac{18816}{55691} \frac{1}{e^{2} \sqrt{1-e^{2}}}\left[\sqrt{1-e^{2}}\left(1-\frac{11 e^{2}}{7}\right) \varphi(e)-\left(1-\frac{3}{7} e^{2}\right) \tilde{\varphi}(e)\right]+\frac{16382 \sqrt{1-e^{2}}}{55691} \frac{\left.\sqrt{1-e^{2}} \psi(e)-\tilde{\psi}(e)\right],}{e^{2}}, \mathrm{~B} 6{ }^{2} \\
& \zeta_{e}(e)=\frac{924}{19067} \frac{1}{e^{2} \sqrt{1-e^{2}}}\left[-\left(1-e^{2}\right)^{3 / 2} \varphi(e)+\left(1-\frac{5}{11} e^{2}\right) \tilde{\varphi}(e)\right]+\frac{12243}{76268} \frac{\sqrt{1-e^{2}}}{e^{2}}\left[\sqrt{1-e^{2}} \zeta(e)-\tilde{\zeta}(e)\right], \\
& \kappa_{e}(e)=\frac{\sqrt{1-e^{2}}}{e^{2}}\left[\sqrt{1-e^{2}} \kappa(e)-\tilde{\kappa}(e)\right]\left(\frac{769}{96}-\frac{3059665}{700566} \ln 2+\frac{8190315}{1868176} \ln 3\right)^{-1}, \\
& F_{e}(e)=\frac{96}{769} \frac{\sqrt{1-e^{2}}}{e^{2}}\left[\sqrt{1-e^{2}} F(e)-\tilde{F}(e)\right] .
\end{aligned}
$$

The eccentricity enhancement functions arise from hereditary contributions to the energy flux (nontilde) and the angular momentum flux (tilde). Most of them do not admit closed forms and have to be computed numerically or in a small-eccentricity expansion. Here we list them in an eccentricity expansion to $\mathcal{O}\left(e^{6}\right)$ :

$$
\begin{aligned}
& \varphi(e)=1+\frac{2335}{192} e^{2}+\frac{42955}{768} e^{4}+\frac{6204647}{36864} e^{6}, \\
& \tilde{\varphi}(e)=1+\frac{209}{32} e^{2}+\frac{2415}{128} e^{4}+\frac{730751}{18432} e^{6} \\
& \psi(e)=1-\frac{22988}{8191} e^{2}-\frac{36508643}{524224} e^{4}-\frac{1741390565}{4718016} e^{6}, \\
& \tilde{\psi}(e)=1-\frac{17416}{8191} e^{2}-\frac{14199197}{524224} e^{4}-\frac{467169215}{4718016} e^{6}, \\
& \kappa(e)=1+e^{2}\left(\frac{62}{3}-\frac{4613840}{350283} \ln 2+\frac{24570945}{1868176} \ln 3\right)+e^{4}\left(\frac{9177}{64}+\frac{271636085}{1401132} \ln 2-\frac{466847955}{7472704} \ln 3\right) \\
& +e^{6}\left(\frac{76615}{128}-\frac{4553279605}{2802264} \ln 2+\frac{14144674005}{119563264} \ln 3+\frac{914306640625}{1076069376} \ln 5\right) \\
& \tilde{\kappa}(e)=1+e^{2}\left(\frac{389}{32}-\frac{2056005}{233522} \ln 2+\frac{8190315}{934088} \ln 3\right)+e^{4}\left(\frac{3577}{64}+\frac{50149295}{467044} \ln 2-\frac{155615985}{3736352} \ln 3\right) \\
& +e^{6}\left(\frac{43049}{256}-\frac{12561332945}{16813584} \ln 2+\frac{4709431125}{59781632} \ln 3+\frac{182861328125}{538034688} \ln 5\right) \text {, } \\
& \zeta(e)=1+\frac{1011565}{48972} e^{2}+\frac{106573021}{783552} e^{4}+\frac{456977827}{854784} e^{6}, \\
& \tilde{\zeta}(e)=1+\frac{102371}{8162} e^{2}+\frac{14250725}{261184} e^{4}+\frac{722230667}{4701312} e^{6}, \\
& F(e)=1+\frac{62}{3} e^{2}+\frac{9177}{64} e^{4}+\frac{76615}{128} e^{6},
\end{aligned}
$$




$$
\tilde{F}(e)=1+\frac{389}{32} e^{2}+\frac{3577}{64} e^{4}+\frac{43049}{256} e^{6} .
$$

By dividing the evolution equations for $x$ and $e$ and expanding in these variables, we can find a solution for the evolution of $x$ in terms of $e$ at each order as described in Sec. III B. Here we provide $x(e)$ at 3PN and to leading order in eccentricity:

$$
x(e)=x_{\mathrm{Newt}}+x_{1 \mathrm{PN}}+x_{1.5 \mathrm{PN}}+x_{2 \mathrm{PN}}+x_{2.5 \mathrm{PN}}+x_{3 \mathrm{PN}},
$$

where

$$
\begin{aligned}
& x_{\text {Newt }}=x_{0}\left[\left(\frac{e_{0}}{e}\right)^{12 / 19}\right] \\
& x_{1 \mathrm{PN}}=x_{0}^{2}\left[\left(\frac{e_{0}}{e}\right)^{24 / 19}\left(-\frac{2833}{3192}+\frac{197 \nu}{114}\right)+\left(\frac{e_{0}}{e}\right)^{12 / 19}\left(\frac{2833}{3192}-\frac{197 \nu}{114}\right)\right] \text {, } \\
& x_{1.5 \mathrm{PN}}=x_{0}^{5 / 2}\left[\frac{377 \pi}{228}\left(\frac{e_{0}}{e}\right)^{12 / 19}-\frac{377 \pi}{228}\left(\frac{e_{0}}{e}\right)^{30 / 19}\right] \text {, } \\
& x_{2 \mathrm{PN}}=x_{0}^{3}\left[\left(\frac{e_{0}}{e}\right)^{12 / 19}\left(-\frac{358353209}{366799104}+\frac{738407 \nu}{727776}+\frac{20597 \nu^{2}}{17328}\right)+\left(\frac{e_{0}}{e}\right)^{24 / 19}\left(-\frac{8025889}{5094432}+\frac{558101 \nu}{90972}-\frac{38809 \nu^{2}}{6498}\right)\right. \\
& \left.+\left(\frac{e_{0}}{e}\right)^{36 / 19}\left(\frac{936217217}{366799104}-\frac{578135 \nu}{80864}+\frac{248681 \nu^{2}}{51984}\right)\right] \\
& x_{2.5 \mathrm{PN}}=x_{0}^{7 / 2}\left[\left(\frac{e_{0}}{e}\right)^{12 / 19}\left(-\frac{3763903 \pi}{7277760}-\frac{12788779 \pi \nu}{1819440}\right)+\left(\frac{e_{0}}{e}\right)^{24 / 19}\left(-\frac{1068041 \pi}{363888}+\frac{74269 \pi \nu}{12996}\right)\right. \\
& \left.+\left(\frac{e_{0}}{e}\right)^{30 / 19}\left(-\frac{5340205 \pi}{1455552}+\frac{371345 \pi \nu}{51984}\right)+\left(\frac{e_{0}}{e}\right)^{42 / 19}\left(\frac{12956437 \pi}{1819440}-\frac{2651489 \pi \nu}{454860}\right)\right], \\
& x_{3 \mathrm{PN}}=x_{0}^{4}\left\{( \frac { e _ { 0 } } { e } ) ^ { 1 2 / 1 9 } \left[\frac{4942027570449143}{96592876047360}+\frac{81025 \pi^{2}}{103968}-\frac{3317 \gamma_{E}}{399}-\frac{12091 \ln 2}{5985}-\frac{78003 \ln 3}{5320}-\frac{3317 \ln x_{0}}{798}\right.\right. \\
& \left.+\left(\frac{10309531979}{7466981760}-\frac{3977 \pi^{2}}{3648}\right) \nu-\frac{267351733 \nu^{2}}{82966464}-\frac{772583 \nu^{3}}{2222316}\right]+\left(\frac{e_{0}}{e}\right)^{30 / 19}\left(-\frac{710645 \pi^{2}}{103968}\right) \\
& +\left(\frac{e_{0}}{e}\right)^{24 / 19}\left(\frac{605942457431}{585411369984}-\frac{3267214507 \nu}{2986792704}-\frac{543796927 \nu^{2}}{82966464}+\frac{27463573 \nu^{3}}{2963088}\right) \\
& +\left(\frac{e_{0}}{e}\right)^{36 / 19}\left(\frac{2652303375761}{390274246656}-\frac{449767537459 \nu}{13938365952}+\frac{2754579983 \nu^{2}}{55310976}-\frac{48990157 \nu^{3}}{1975392}\right) \\
& +\left(\frac{e_{0}}{e}\right)^{48 / 19}\left[-\frac{1628129474693173}{27597964584960}+\frac{157405 \pi^{2}}{25992}+\frac{3317 \gamma_{E}}{399}+\frac{12091 \ln 2}{5985}+\frac{78003 \ln 3}{5320}+\frac{6634}{2527} \ln \left(\frac{e_{0}}{e}\right)\right. \\
& \left.\left.+\frac{3317 \ln x_{0}}{798}+\left(\frac{6686551181963}{209075489280}+\frac{3977 \pi^{2}}{3648}\right) \nu-\frac{6641442629 \nu^{2}}{165932928}+\frac{282310639 \nu^{3}}{17778528}\right]\right\} \text {. }
\end{aligned}
$$

\section{APPENDIX C: OSCILLATORY MEMORY INTEGRAL}

Here we derive the formula to evaluate the oscillatory memory integrals in Eq. (30). For convenience we set $G=c=1$ in this Appendix. We define the integral that has to be computed as

$$
J_{\mathrm{mem}}=\int_{-\infty}^{T_{R}} \mathrm{~d} t x^{p}(t) e^{q}(t) \mathrm{e}^{\mathrm{i}\left(s \lambda_{\xi}+r \xi\right)}
$$


We follow the approach of Ref. [73], where this integral was evaluated in the case of circular orbits $(q=0)$. The eccentric orbit is assumed to evolve only with the secular radiation-reaction equations given in Eqs. (15a)-(15b) starting from $x=0$ and $e=1$ in the remote past. Every astrophysical process like capture or mass loss possibly happening to the binary is ignored. We start by restating the evolution equation for $x$ at leading order in $x$ and $e$,

$$
\frac{\mathrm{d} x(t)}{\mathrm{d} t}=\frac{64 \nu x^{5}(t)}{5 m}\left[1+\frac{157}{24} e^{2}(t)\right]
$$

and integrate it over a time interval up to some coalescence time $T_{C}$, where the orbital frequency and therefore $x$ tends to infinity:

$$
\int_{t}^{T_{C}} \mathrm{~d} t=\int_{x(t)}^{\infty} \frac{\mathrm{d} x(t)}{(\mathrm{d} x / \mathrm{d} t)}
$$

Thereby, we find an explicit relation between the orbital frequency (related to $x$ ) and time $t$ :

$$
T_{C}-t=\frac{5 m}{256 \nu} \frac{1}{x^{4}(t)}\left[1-\frac{157}{43} e^{2}(t)\right] .
$$

We can now invert the $x(e)$ relation derived in Eq. (25) to find $e$ as a function of $x$. Considering only the leading order, we find

$$
e(t)=e\left(T_{R}\right)\left(\frac{x\left(T_{R}\right)}{x(t)}\right)^{19 / 12} .
$$

Using Eqs. (C4) and (C5), we get $x$ as an explicit function of $t$ :

$x(t)=\frac{1}{4}\left(\frac{5 m}{\nu\left(T_{C}-t\right)}\right)^{1 / 4}\left[1-\frac{157}{172} e^{2}\left(T_{R}\right)\left(\frac{T_{C}-t}{T_{C}-T_{R}}\right)^{19 / 24}\right]$.

A quick check reveals that this expression indeed solves the differential equation in Eq. (C2). Since the memory integral runs up to the current time $T_{R}$, we introduce a new integration variable $y$ which is better suited to the integration limits we have:

$$
y=\frac{T_{R}-t}{T_{C}-T_{R}} .
$$

Next, we express the time-dependent quantities in the integral in terms of $y$ and their values at the current time $T_{R}$. For $x$ we find

$x(y)=x\left(T_{R}\right)(1+y)^{-1 / 4}\left[1-\frac{157}{172} e^{2}\left(T_{R}\right)\left((1+y)^{19 / 24}-1\right)\right]$,

and for the eccentricity we find

$$
e(y)=e\left(T_{R}\right)(1+y)^{19 / 48} .
$$

Note that while going back in time, with increasing $y$, we only let the eccentricity evolve until $e=1$ is reached. Furthermore, we need the redefined mean anomaly $\xi(t)$ in terms of $y$ and its value at the current time. Because $\xi$ is defined in terms of $\dot{\xi}=n$, we have to calculate the integral

$$
\begin{aligned}
\xi(t) & =\xi\left(T_{C}\right)-\int_{T_{C}}^{t} \mathrm{~d} t^{\prime} n\left(t^{\prime}\right) \\
& =\xi\left(T_{C}\right)-\frac{1}{m} \int_{T_{C}}^{t} \mathrm{~d} t^{\prime} x^{3 / 2}\left(t^{\prime}\right) \\
& =\xi\left(T_{C}\right)-\frac{\left(T_{C}-T_{R}\right)}{m} \int_{-1}^{y} \mathrm{~d} y^{\prime} x^{3 / 2}\left(y^{\prime}\right) .
\end{aligned}
$$

We can now evaluate the latter integral by inserting the expression for $x(y)$ given in Eq. (C8). This leads to

$$
\begin{aligned}
\xi(t)= & \xi\left(T_{C}\right)-\frac{8\left(T_{C}-T_{R}\right) x^{3 / 2}\left(T_{R}\right)}{5 m}(1+y)^{5 / 8} \\
& \times\left[1-\frac{471}{11696} e^{2}\left(T_{R}\right)\left(15(1+y)^{19 / 24}-34\right)\right],
\end{aligned}
$$

where $\xi\left(T_{C}\right)$ is the value of $\xi$ at the moment of coalescence. Thus, at the current time $T_{R}$ the mean anomaly is given by

$$
\xi\left(T_{R}\right)=\xi\left(T_{C}\right)-\frac{8\left(T_{C}-T_{R}\right) x^{3 / 2}\left(T_{R}\right)}{5 m}\left[1+\frac{8949}{11696} e^{2}\left(T_{R}\right)\right]
$$

Now we are able to express $\xi(t)$ in terms of $\xi\left(T_{R}\right)$ and $y$,

$$
\xi(t)=\xi\left(T_{R}\right)-\frac{8\left(T_{C}-T_{R}\right) x^{3 / 2}\left(T_{R}\right)}{5 m}\left[(1+y)^{5 / 8}-1\right]\left[1-\frac{471}{11696} e^{2}\left(T_{R}\right) \frac{19-34(1+y)^{5 / 8}+15(1+y)^{17 / 12}}{(1+y)^{5 / 8}-1}\right],
$$

where $x\left(T_{R}\right)$ and $e\left(T_{R}\right)$ stand for the respective current values of $x$ and $e$. 
At this point, we introduce a dimensionless "adiabatic parameter" $\chi\left(T_{R}\right)$, which is connected with the inspiral rate at the current retarded time $T_{R}$. We define it as the ratio between the current period and the time left until coalescence,

$$
\chi\left(T_{R}\right)=\frac{1}{n\left(T_{R}\right)\left(T_{C}-T_{R}\right)},
$$

where $n\left(T_{R}\right)=x^{3 / 2}\left(T_{R}\right) / m$ at leading order. Explicitly in terms of $x\left(T_{R}\right)$ and $e\left(T_{R}\right)$, it reads

$$
\chi\left(T_{R}\right)=\frac{256 \nu}{5} x^{5 / 2}\left(T_{R}\right)\left[1+\frac{157}{43} e^{2}\left(T_{R}\right)\right] .
$$

Inserting $\chi\left(T_{R}\right)$ into Eq. (C13), we find

$$
\xi(t)=\xi\left(T_{R}\right)-\frac{8}{5 \chi\left(T_{R}\right)}\left[(1+y)^{5 / 8}-1\right]\left[1-\frac{471}{11696} e^{2}\left(T_{R}\right) \frac{19-34(1+y)^{5 / 8}+15(1+y)^{17 / 12}}{(1+y)^{5 / 8}-1}\right] .
$$

Now we put Eqs. (C8), (C9), and (C16) into the oscillatory integral and write it as an integral over $y$ :

$$
\begin{aligned}
J_{\mathrm{mem}}= & \left(T_{C}-T_{R}\right) \int_{0}^{\infty} \mathrm{d} y x^{p}(y) e^{q}(y) \mathrm{e}^{\mathrm{i}\left(s \lambda_{\xi}(y)+r \xi(y)\right)} \\
= & \left(T_{C}-T_{R}\right) \mathrm{e}^{\mathrm{i}(r+s(1+k)) \xi\left(T_{R}\right)} \int_{0}^{\infty} \mathrm{d} y x^{p}(y) e^{q}(y) \exp \left\{-\frac{8 \mathrm{i}(r+s(1+k))}{5 \chi\left(T_{R}\right)}\left[(1+y)^{5 / 8}-1\right]\right. \\
& \left.\times\left[1-\frac{471}{11696} e^{2}\left(T_{R}\right) \frac{19-34(1+y)^{5 / 8}+15(1+y)^{17 / 12}}{(1+y)^{5 / 8}-1}\right]\right\} .
\end{aligned}
$$

Let us look at the form of this integral:

$$
J_{\mathrm{mem}} \sim \int_{0}^{\infty} \mathrm{d} y f(y) \exp \left[\frac{\mathrm{i}}{\chi\left(T_{R}\right)} g(y)\right]
$$

The strategy is to integrate by parts, and therefore we need to know the following type of integral:

$$
\int \mathrm{d} y \mathrm{e}^{\mathrm{i} \sigma g(y)}=-\frac{\mathrm{i}}{\sigma g^{\prime}(y)} \mathrm{e}^{\mathrm{i} \sigma g(y)}+\mathcal{O}\left(g^{\prime}(y)^{-2}\right) .
$$

This formula is valid as long as $g^{\prime}(y)$ is sufficiently large. Integrating Eq. (C18) by parts, we get

$$
J_{\text {mem }} \sim f(y)\left[-\frac{\mathrm{i} \chi\left(T_{R}\right)}{g^{\prime}(y)} \exp \left[\frac{\mathrm{i}}{\chi\left(T_{R}\right)} g(y)\right]\right]_{0}^{\infty}+\mathrm{i} \chi\left(T_{R}\right) \int_{0}^{\infty} \mathrm{d} y \frac{f^{\prime}(y)}{g^{\prime}(y)} \exp \left[\frac{\mathrm{i}}{\chi\left(T_{R}\right)} g(y)\right] .
$$

As $y$ approaches infinity in the remote past, we notice that $f(y)=x^{p}(y) e^{q}(y)$ goes to zero. This is because at early times the frequency reaches zero and the eccentricity cannot grow past $e=1$ in our model. Evaluating the first term at $y=0$, we recover $x$ and $e$ at the current time and the exponential factor is just 1 since $g(0)=0$. The derivative $g^{\prime}(y)$ in the denominator evaluated at $y=0$ is effectively 1 multiplied by some constants. What remains in the first term of Eq. (C20) is therefore of order $\chi\left(T_{R}\right)$. Looking at the second term, we find the same integral form as in Eq. (C18). Successively integrating by parts would yield another factor of $\chi\left(T_{R}\right)$ each time. Since this parameter is already of order 2.5PN, the higher-order $\chi\left(T_{R}\right)$ contributions can be safely ignored. Including everything of order $\chi\left(T_{R}\right)$, we find the formula

$$
\begin{aligned}
J_{\text {mem }} & =-\left(T_{c}-T_{R}\right) x^{p} e^{q} \mathrm{e}^{\mathrm{i}\left(s \lambda_{\xi}+r_{\xi}\right)} \frac{\mathrm{i} \chi\left(T_{R}\right)}{(r+s(1+k))} \\
& =-\frac{\mathrm{i}}{n(r+s(1+k))} x^{p} e^{q} \mathrm{e}^{\mathrm{i}\left(s \lambda_{\xi}+r \xi\right)},
\end{aligned}
$$

which allows us to compute the oscillatory hereditary integrals in Sec. III C. 


\section{APPENDIX D: LIST OF DC MEMORY MODES}

Here we list the $3 \mathrm{PN}$-accurate DC memory contributions to the $h^{\ell m}$ modes at leading order in eccentricity in the following form:

$$
h_{\mathrm{DC}}^{\ell m}=\frac{8 G m \nu}{c^{2} R} x \sqrt{\frac{\pi}{5}} H_{\mathrm{DC}}^{\ell m}
$$

where $H_{\mathrm{DC}}^{\ell m}$ is a function of $x$ and $e$. The nonzero modes read

$$
\begin{aligned}
& H_{\mathrm{DC}}^{20}=-\frac{5}{14 \sqrt{6}}\left(H_{\mathrm{Newt}}^{20}+x H_{1 \mathrm{PN}}^{20}+x^{3 / 2} H_{1.5 \mathrm{PN}}^{20}+x^{2} H_{2 \mathrm{PN}}^{20}+x^{5 / 2} H_{2.5 \mathrm{PN}}^{20}+x^{3} H_{3 \mathrm{PN}}^{20}\right), \\
& H_{\text {Newt }}^{20}=1-\left(\frac{e}{e_{i}}\right)^{12 / 19} \\
& H_{1 \mathrm{PN}}^{20}=-\frac{4075}{4032}+\frac{67 \nu}{48}+\left(\frac{e}{e_{i}}\right)^{12 / 19}\left(-\frac{2833}{3192}+\frac{197 \nu}{114}\right)+\left(\frac{e}{e_{i}}\right)^{24 / 19}\left(\frac{145417}{76608}-\frac{2849 \nu}{912}\right), \\
& H_{1.5 \mathrm{PN}}^{20}=-\frac{377 \pi}{228}\left(\frac{e}{e_{i}}\right)^{12 / 19}+\frac{377 \pi}{228}\left(\frac{e}{e_{i}}\right)^{30 / 19} \\
& H_{2 \mathrm{PN}}^{20}=-\frac{151877213}{67060224}-\frac{123815 \nu}{44352}+\frac{205 \nu^{2}}{352}+\left(\frac{e}{e_{i}}\right)^{12 / 19}\left(\frac{358353209}{366799104}-\frac{738407 \nu}{727776}-\frac{20597 \nu^{2}}{17328}\right) \\
& +\left(\frac{e}{e_{i}}\right)^{24 / 19}\left(\frac{411966361}{122266368}-\frac{825950 \nu}{68229}+\frac{561253 \nu^{2}}{51984}\right) \\
& +\left(\frac{e}{e_{i}}\right)^{36 / 19}\left(-\frac{50392977379}{24208740864}+\frac{764295307 \nu}{48033216}-\frac{11654209 \nu^{2}}{1143648}\right) \text {, } \\
& H_{2.5 \mathrm{PN}}^{20}=-\frac{253 \pi}{336}+\frac{253 \pi \nu}{84}+\left(\frac{e}{e_{i}}\right)^{12 / 19}\left(\frac{3763903 \pi}{7277760}+\frac{12788779 \pi \nu}{1819440}\right)+\left(\frac{e}{e_{i}}\right)^{24 / 19}\left(\frac{54822209 \pi}{8733312}-\frac{1074073 \pi \nu}{103968}\right) \\
& +\left(\frac{e}{e_{i}}\right)^{30 / 19}\left(\frac{5340205 \pi}{1455552}-\frac{371345 \pi \nu}{51984}\right)+\left(\frac{e}{e_{i}}\right)^{42 / 19}\left(-\frac{424020733 \pi}{43666560}+\frac{27049187 \pi \nu}{3638880}\right), \\
& H_{3 \mathrm{PN}}^{20}=-\frac{4397711103307}{532580106240}+\left(\frac{700464542023}{13948526592}-\frac{205 \pi^{2}}{96}\right) \nu+\frac{69527951 \nu^{2}}{166053888}+\frac{1321981 \nu^{3}}{5930496} \\
& +\left(\frac{e}{e_{i}}\right)^{12 / 19}\left[-\frac{4942027570449143}{96592876047360}-\frac{81025 \pi^{2}}{103968}+\frac{3317 \gamma_{E}}{399}+\left(-\frac{10309531979}{7466981760}+\frac{3977 \pi^{2}}{3648}\right) \nu\right. \\
& \left.+\frac{267351733 \nu^{2}}{82966464}+\frac{772583 \nu^{3}}{2222316}+\frac{12091 \ln 2}{5985}+\frac{78003 \ln 3}{5320}+\frac{3317 \ln x}{798}\right]+\frac{710645 \pi^{2}}{103968}\left(\frac{e}{e_{i}}\right)^{30 / 19} \\
& +\left(\frac{e}{e_{i}}\right)^{24 / 19}\left(-\frac{31102835980319}{14049872879616}+\frac{279737759653 \nu}{167260391424}+\frac{26730466283 \nu^{2}}{1991195136}-\frac{397176241 \nu^{3}}{23704704}\right) \\
& +\left(\frac{e}{e_{i}}\right)^{36 / 19}\left(-\frac{142763304914707}{25758100279296}+\frac{48901891428821 \nu}{919932152832}-\frac{400181473249 \nu^{2}}{3650524416}+\frac{2295879173 \nu^{3}}{43458624}\right) \\
& +\left(\frac{e}{e_{i}}\right)^{48 / 19}\left[\frac{385621605844415513}{5740376633671680}-\frac{157405 \pi^{2}}{25992}-\frac{3317 \gamma_{E}}{399}+\left(-\frac{49590995147570629}{478364719472640}+\frac{1271 \pi^{2}}{1216}\right) \nu\right. \\
& \left.+\frac{3194536246463 \nu^{2}}{34514049024}-\frac{1672948713 \nu^{3}}{45653504}-\frac{12091 \ln 2}{5985}-\frac{78003 \ln 3}{5320}-\frac{3317 \ln x}{798}-\frac{6634}{2527} \ln \left(\frac{e}{e_{i}}\right)\right],
\end{aligned}
$$




$$
\begin{aligned}
& H_{\mathrm{DC}}^{40}=-\frac{1}{504 \sqrt{2}}\left(H_{\mathrm{Newt}}^{40}+x H_{1 \mathrm{PN}}^{40}+x^{3 / 2} H_{1.5 \mathrm{PN}}^{40}+x^{2} H_{2 \mathrm{PN}}^{40}+x^{5 / 2} H_{2.5 \mathrm{PN}}^{40}+x^{3} H_{3 \mathrm{PN}}^{40}\right), \\
& H_{\text {Newt }}^{40}=1-\left(\frac{e}{e_{i}}\right)^{12 / 19} \\
& H_{1 \mathrm{PN}}^{40}=-\frac{180101}{29568}+\frac{27227 \nu}{1056}+\left(\frac{e}{e_{i}}\right)^{12 / 19}\left(-\frac{2833}{3192}+\frac{197 \nu}{114}\right)+\left(\frac{e}{e_{i}}\right)^{24 / 19}\left(\frac{3920527}{561792}-\frac{183995 \nu}{6688}\right), \\
& H_{1.5 \mathrm{PN}}^{40}=-\frac{377 \pi}{228}\left(\frac{e}{e_{i}}\right)^{12 / 19}+\frac{377 \pi}{228}\left(\frac{e}{e_{i}}\right)^{30 / 19} \\
& H_{2 \mathrm{PN}}^{40}=\frac{2201411267}{158505984}-\frac{34829479 \nu}{432432}+\frac{844951 \nu^{2}}{27456}+\left(\frac{e}{e_{i}}\right)^{12 / 19}\left(\frac{358353209}{366799104}-\frac{738407 \nu}{727776}-\frac{20597 \nu^{2}}{17328}\right) \\
& +\left(\frac{e}{e_{i}}\right)^{24 / 19}\left(\frac{11106852991}{896620032}-\frac{584029331 \nu}{8005536}+\frac{36247015 \nu^{2}}{381216}\right) \\
& +\left(\frac{e}{e_{i}}\right)^{36 / 19}\left(-\frac{17153749047583}{629427262464}+\frac{24120402175 \nu}{156107952}-\frac{1235668217 \nu^{2}}{9911616}\right), \\
& H_{2.5 \mathrm{PN}}^{40}=-\frac{13565 \pi}{1232}+\frac{13565 \pi \nu}{308}+\left(\frac{e}{e_{i}}\right)^{12 / 19}\left(\frac{3763903 \pi}{7277760}+\frac{12788779 \pi \nu}{1819440}\right) \\
& +\left(\frac{e}{e_{i}}\right)^{24 / 19}\left(\frac{1478038679 \pi}{64044288}-\frac{69366115 \pi \nu}{762432}\right)+\left(\frac{e}{e_{i}}\right)^{30 / 19}\left(\frac{5340205 \pi}{1455552}-\frac{371345 \pi \nu}{51984}\right) \\
& +\left(\frac{e}{e_{i}}\right)^{42 / 19}\left(-\frac{473166857 \pi}{29111040}+\frac{1255597433 \pi \nu}{26685120}\right) \text {, }
\end{aligned}
$$$$
H_{3 \mathrm{PN}}^{40}=\frac{15240463356751}{781117489152}+\left(-\frac{1029744557245}{27897053184}-\frac{205 \pi^{2}}{96}\right) \nu-\frac{4174614175 \nu^{2}}{36900864}+\frac{221405645 \nu^{3}}{11860992}
$$$$
+\left(\frac{e}{e_{i}}\right)^{12 / 19}\left[-\frac{4942027570449143}{96592876047360}-\frac{81025 \pi^{2}}{103968}+\frac{3317 \gamma_{E}}{399}+\left(-\frac{10309531979}{7466981760}+\frac{3977 \pi^{2}}{3648}\right) \nu\right.
$$$$
\left.+\frac{267351733 \nu^{2}}{82966464}+\frac{772583 \nu^{3}}{2222316}+\frac{12091 \ln 2}{5985}+\frac{78003 \ln 3}{5320}+\frac{3317 \ln x}{798}\right]+\frac{710645 \pi^{2}}{103968}\left(\frac{e}{e_{i}}\right)^{30 / 19}
$$$$
+\left(\frac{e}{e_{i}}\right)^{24 / 19}\left(-\frac{838550569998089}{103032401117184}+\frac{30467243664175 \nu}{1226576203776}+\frac{963631094693 \nu^{2}}{14602097664}-\frac{25650558955 \nu^{3}}{173834496}\right)
$$$$
+\left(\frac{e}{e_{i}}\right)^{36 / 19}\left(-\frac{48596571051802639}{669710607261696}+\frac{13219254870469451 \nu}{23918235973632}-\frac{107533340184449 \nu^{2}}{94913634816}+\frac{243426638749 \nu^{3}}{376641408}\right)
$$$$
+\left(\frac{e}{e_{i}}\right)^{48 / 19}\left[\frac{1289915690995598063}{11480753267343360}-\frac{157405 \pi^{2}}{25992}-\frac{3317 \gamma_{E}}{399}+\left(-\frac{515898615572711953}{956729438945280}+\frac{1271 \pi^{2}}{1216}\right) \nu\right.
$$$$
\left.+\frac{297870712456705 \nu^{2}}{253103026176}-\frac{520032054523 \nu^{3}}{1004377088}-\frac{12091 \ln 2}{5985}-\frac{78003 \ln 3}{5320}-\frac{3317 \ln x}{798}-\frac{6634}{2527} \ln \left(\frac{e}{e_{i}}\right)\right] \text {, }
$$$$
H_{\mathrm{DC}}^{60}=\frac{4195}{1419264 \sqrt{273}}\left(x H_{1 \mathrm{PN}}^{60}+x^{2} H_{2 \mathrm{PN}}^{60}+x^{5 / 2} H_{2.5 \mathrm{PN}}^{60}+x^{3} H_{3 \mathrm{PN}}^{60}\right)
$$$$
H_{1 \mathrm{PN}}^{60}=1-\frac{3612 \nu}{839}
$$ 


$$
\begin{aligned}
& H_{2 \mathrm{PN}}^{60}=-\frac{45661561}{6342840}+\frac{101414 \nu}{2517}-\frac{48118 \nu^{2}}{839}+\left(\frac{e}{e_{i}}\right)^{24 / 19}\left(-\frac{2833}{1596}+\frac{530740 \nu}{47823}-\frac{237188 \nu^{2}}{15941}\right) \\
& +\left(\frac{e}{e_{i}}\right)^{36 / 19}\left(\frac{1081489489}{120513960}-\frac{819202 \nu}{15941}+\frac{1151430 \nu^{2}}{15941}\right) \text {, } \\
& H_{2.5 \mathrm{PN}}^{60}=\frac{1248 \pi}{839}-\frac{4992 \pi \nu}{839}+\left(\frac{e}{e_{i}}\right)^{24 / 19}\left(-\frac{377 \pi}{114}+\frac{226954 \pi \nu}{15941}\right)+\left(\frac{e}{e_{i}}\right)^{42 / 19}\left(\frac{174031 \pi}{95646}-\frac{132106 \pi \nu}{15941}\right), \\
& H_{3 \mathrm{PN}}^{60}=\frac{3012132889099}{144921208320}-\frac{27653500031 \nu}{191694720}+\frac{1317967427 \nu^{2}}{4107744}-\frac{24793657 \nu^{3}}{342312} \\
& +\left(\frac{e}{e_{i}}\right)^{24 / 19}\left(\frac{213887207}{183399552}-\frac{7295329871 \nu}{1831812192}-\frac{214435261 \nu^{2}}{21807288}+\frac{41962109 \nu^{3}}{1817274}\right) \\
& +\left(\frac{e}{e_{i}}\right)^{36 / 19}\left(\frac{3063859722337}{128226853440}-\frac{839669231153 \nu}{4579530480}+\frac{555765673 \nu^{2}}{1211516}-\frac{113415855 \nu^{3}}{302879}\right) \\
& +\left(\frac{e}{e_{i}}\right)^{48 / 19}\left(-\frac{9789584507539}{213536964096}+\frac{206521649193667 \nu}{622816145280}-\frac{380487275717 \nu^{2}}{494298528}+\frac{17456918535 \nu^{3}}{41191544}\right), \\
& H_{\mathrm{DC}}^{80}=-\frac{75601}{213497856 \sqrt{119}}\left(x^{2} H_{2 \mathrm{PN}}^{80}+x^{3} H_{3 \mathrm{PN}}^{80}\right) \\
& H_{2 \mathrm{PN}}^{80}=1-\frac{452070 \nu}{75601}+\frac{733320 \nu^{2}}{75601}+\left(\frac{e}{e_{i}}\right)^{36 / 19}\left(-1+\frac{452070 \nu}{75601}-\frac{733320 \nu^{2}}{75601}\right) \text {, } \\
& H_{3 \mathrm{PN}}^{80}=-\frac{265361599}{33869248}+\frac{18177898147 \nu}{321757856}-\frac{722521125 \nu^{2}}{5745676}+\frac{261283995 \nu^{3}}{2872838} \\
& +\left(\frac{e}{e_{i}}\right)^{36 / 19}\left(-\frac{2833}{1064}+\frac{848864713 \nu}{40219732}-\frac{81627030 \nu^{2}}{1436419}+\frac{72232020 \nu^{3}}{1436419}\right) \\
& +\left(\frac{e}{e_{i}}\right)^{48 / 19}\left(\frac{50791665}{4838464}-\frac{3566973693 \nu}{45965408}+\frac{1049029245 \nu^{2}}{5745676}-\frac{405748035 \nu^{3}}{2872838}\right), \\
& H_{\mathrm{DC}}^{100}=\frac{525221}{6452379648 \sqrt{154}} x^{3}\left[1-\frac{79841784 \nu}{9979199}+\frac{198570240 \nu^{2}}{9979199}-\frac{172307520 \nu^{3}}{9979199}\right. \\
& \left.+\left(\frac{e}{e_{i}}\right)^{48 / 19}\left(-1+\frac{79841784 \nu}{9979199}-\frac{198570240 \nu^{2}}{9979199}+\frac{172307520 \nu^{3}}{9979199}\right)\right] .
\end{aligned}
$$

\section{APPENDIX E: LIST OF OSCILLATORY MEMORY MODES}

Here we list the nonzero oscillatory memory contributions to the $h^{\ell m}$ modes at $3 \mathrm{PN}$ order and to quadratic order in eccentricity in the following way:

$$
h_{\mathrm{osc}}^{\ell m}=\frac{8 G m \nu}{c^{2} R} x \sqrt{\frac{\pi}{5}} \mathrm{e}^{-\mathrm{i} m \psi} H_{\mathrm{osc}}^{\ell m},
$$

where $H_{\mathrm{osc}}^{\ell m}$ is a function of $x, e$, and the modified mean anomaly $\xi$. To improve readability in the odd- $m$ expressions, we define $\Delta=\left(m_{1}-m_{2}\right) / m=\sqrt{1-4 \nu}$ : 


$$
\begin{aligned}
& H_{\mathrm{osc}}^{20}=\frac{\sqrt{6} \mathrm{i} x^{5 / 2} e \nu}{7}\left[-16 \mathrm{e}^{-\mathrm{i} \xi}+16 \mathrm{e}^{\mathrm{i} \xi}-\frac{647}{36} e \mathrm{e}^{-2 \mathrm{i} \xi}+\frac{647}{36} e \mathrm{e}^{2 \mathrm{i} \xi}\right] \\
& H_{\mathrm{osc}}^{22}=\mathrm{i} x^{3 / 2} e^{2} \nu \mathrm{e}^{2 \mathrm{i} \xi}\left[-\frac{13}{252}+\left(\frac{697}{336}-\frac{865 \nu}{216}\right) x-\frac{29 \pi}{126} x^{3 / 2}\right]+\frac{\mathrm{i} x^{5 / 2} e \nu}{21}\left[\frac{19}{6} e+\frac{4}{3} \mathrm{e}^{-\mathrm{i} \xi}-4 \mathrm{e}^{\mathrm{i} \xi}+\frac{65}{24} e \mathrm{e}^{-2 \mathrm{i} \xi}\right] \\
& H_{\mathrm{osc}}^{31}=\frac{\sqrt{14} x^{2} \nu \Delta}{90}\left\{\frac{44}{3} e^{2}-\frac{44}{3} e \mathrm{e}^{\mathrm{i} \xi}-\frac{44}{3} e^{2} \mathrm{e}^{2 \mathrm{i} \xi}+x\left[-\frac{121}{7}-\frac{43}{2} e \mathrm{e}^{-\mathrm{i} \xi}-\frac{2987}{84} e^{2} \mathrm{e}^{-2 \mathrm{i} \xi}\right.\right. \\
& \left.\left.+e \mathrm{e}^{\mathrm{i} \xi}\left(\frac{19801}{264}-\frac{2521 \nu}{231}\right)+e^{2} \mathrm{e}^{2 \mathrm{i} \xi}\left(\frac{7957}{88}-\frac{827 \nu}{231}\right)+e^{2}\left(-\frac{111821}{616}+\frac{827 \nu}{231}\right)\right]\right\}, \\
& H_{\mathrm{osc}}^{33}=\frac{x^{3} \nu \Delta}{6 \sqrt{210}}\left[\frac{22}{9}+19 e^{2}+11 e \mathrm{e}^{-\mathrm{i} \xi}+\frac{1}{3} e \mathrm{e}^{\mathrm{i} \xi}+\frac{713}{30} e^{2} \mathrm{e}^{-2 \mathrm{i} \xi}-\frac{119}{6} e^{2} \mathrm{e}^{2 \mathrm{i} \xi}\right], \\
& H_{\text {osc }}^{40}=\frac{\sqrt{2} \mathrm{i} x^{5 / 2} e \nu}{210}\left[-8 \mathrm{e}^{-\mathrm{i} \xi}+8 \mathrm{e}^{\mathrm{i} \xi}-\frac{143}{16} e \mathrm{e}^{-2 \mathrm{i} \xi}+\frac{143}{16} e \mathrm{e}^{2 \mathrm{i} \xi}\right] \\
& H_{\mathrm{osc}}^{42}=\frac{\mathrm{i} x^{3 / 2} e^{2} \nu \mathrm{e}^{2 \mathrm{i} \xi}}{216 \sqrt{5}}\left[-\frac{13}{14}+x\left(\frac{7943}{56}-\frac{25393 \nu}{66}\right)-\frac{29}{7} \pi x^{3 / 2}\right]+\frac{\mathrm{i} x^{5 / 2} e \nu}{126 \sqrt{5}}\left[\frac{19}{12} e+\frac{2}{3} \mathrm{e}^{-\mathrm{i} \xi}-2 \mathrm{e}^{\mathrm{i} \xi}+\frac{65}{48} e \mathrm{e}^{-2 \mathrm{i} \xi}\right] \\
& H_{\mathrm{osc}}^{44}=\frac{\mathrm{i} x^{5 / 2} \nu}{6 \sqrt{35}}\left[\frac{2}{3}+\frac{331 e^{2}}{240}+\frac{14}{15} e \mathrm{e}^{-\mathrm{i} \xi}+2 e \mathrm{e}^{\mathrm{i} \xi}+\frac{1037}{720} e^{2} \mathrm{e}^{-2 \mathrm{i} \xi}+\frac{217}{48} e^{2} \mathrm{e}^{2 \mathrm{i} \xi}\right] \\
& H_{\mathrm{osc}}^{51}=\frac{x^{2} \nu \Delta}{18 \sqrt{385}}\left\{\frac{43 e^{2}}{12}-\frac{43}{12} e \mathrm{e}^{\mathrm{i} \xi}-\frac{43}{12} e^{2} \mathrm{e}^{2 \mathrm{i} \xi}+x\left[-\frac{26}{7}-\frac{251}{56} e \mathrm{e}^{-\mathrm{i} \xi}-\frac{1199}{168} e^{2} \mathrm{e}^{-2 \mathrm{i} \xi}\right.\right. \\
& \left.\left.+e \mathrm{e}^{\mathrm{i} \xi}\left(\frac{8627}{156}-\frac{41807 \nu}{312}\right)+e^{2} \mathrm{e}^{2 \mathrm{i} \xi}\left(\frac{785}{13}-\frac{5156 \nu}{39}\right)+e^{2}\left(-\frac{8321}{104}+\frac{5156 \nu}{39}\right)\right]\right\}, \\
& H_{\mathrm{osc}}^{53}=\frac{x^{3} \nu \Delta}{2 \sqrt{330}}\left[-\frac{2}{189}+\frac{27 e^{2}}{112}+\frac{41}{336} e \mathrm{e}^{-\mathrm{i} \xi}-\frac{67}{504} e \mathrm{e}^{\mathrm{i} \xi}+\frac{1531 e^{2} \mathrm{e}^{-2 \mathrm{i} \xi}}{5040}-\frac{47}{72} e^{2} \mathrm{e}^{2 \mathrm{i} \xi}\right] \\
& H_{\mathrm{osc}}^{55}=\frac{x^{3} \nu \Delta}{14 \sqrt{66}}\left[\frac{18}{5}+\frac{8909 e^{2}}{720}+\frac{457}{72} e \mathrm{e}^{-\mathrm{i} \xi}+\frac{197}{16} e \mathrm{e}^{\mathrm{i} \xi}+\frac{787}{72} e^{2} \mathrm{e}^{-2 \mathrm{i} \xi}+\frac{4369}{144} e^{2} \mathrm{e}^{2 \mathrm{i} \xi}\right] \\
& H_{\mathrm{osc}}^{62}=\frac{\mathrm{i} x^{5 / 2} e^{2} \nu \mathrm{e}^{2 \mathrm{i} \xi}}{352 \sqrt{65}}\left[\frac{2783}{168}-53 \nu\right] \\
& H_{\mathrm{osc}}^{71}=\frac{5 x^{3} \nu e \Delta}{30888 \sqrt{2}}\left[\mathrm{e}^{\mathrm{i} \xi}\left(\frac{5023}{168}-97 \nu\right)+e \mathrm{e}^{2 \mathrm{i} \xi}\left(\frac{5023}{168}-97 \nu\right)+e\left(-\frac{5023}{168}+97 \nu\right)\right] \text {. }
\end{aligned}
$$

[1] J. Aasi et al., Classical Quantum Gravity 32, 115012 (2015).

[2] T. Accadia et al., J. Instrum. 7, P03012 (2012).

[3] H. Grote (LIGO Scientific Collaboration), Classical Quantum Gravity 27, 084003 (2010).

[4] B. P. Abbott et al., Phys. Rev. Lett. 116, 061102 (2016).

[5] B. P. Abbott et al., Phys. Rev. Lett. 116, 241103 (2016).
[6] B. P. Abbott et al., Phys. Rev. Lett. 118, 221101 (2017).

[7] B. P. Abbott et al., Phys. Rev. Lett. 119, 141101 (2017).

[8] B. P. Abbott et al., Phys. Rev. Lett. 119, 161101 (2017).

[9] B. P. Abbott et al., Astrophys. J. 851, L35 (2017).

[10] B. P. Abbott et al. (LIGO Scientific and Virgo Collaborations), Phys. Rev. X 9, 031040 (2019). 
[11] T. Akutsu et al., Nat. Astron. 3, 35 (2019).

[12] B. R. Iyer et al., LIGO-India Technical Report No. LIGOM1100296, 2011, https://dcc.ligo.org/LIGO-M1100296/ public/main.

[13] P. Amaro-Seoane, H. Audley, S. Babak, J. Baker, E. Barausse, P. Bender, E. Berti et al., arXiv:1702.00786.

[14] R. N. Manchester and IPTA, Classical Quantum Gravity 30, 224010 (2013).

[15] J. M. Weisberg and Y. Huang, Astrophys. J. 829, 55 (2016).

[16] P. C. Peters and J. Mathews, Phys. Rev. 131, 435 (1963).

[17] B. Sun, Z. Cao, Y. Wang, and H.-C. Yeh, Phys. Rev. D 92, 044034 (2015).

[18] E. A. Huerta, C. J. Moore, P. Kumar, D. George, A. J. K. Chua, R. Haas, E. Wessel, D. Johnson, D. Glennon, A. Rebei, A. M. Holgado, J. R. Gair, and H. P. Pfeiffer, Phys. Rev. D 97, 024031 (2018).

[19] J. Samsing, Phys. Rev. D 97, 103014 (2018).

[20] J. Samsing, M. MacLeod, and E. Ramirez-Ruiz, Astrophys. J. 784, 71 (2014).

[21] R. M. O'Leary, R. O’Shaughnessy, and F. A. Rasio, Phys. Rev. D 76, 061504 (2007).

[22] S. Naoz, B. Kocsis, A. Loeb, and N. Yunes, Astrophys. J. 773, 187 (2013).

[23] D. Park, C. Kim, H. M. Lee, Y.-B. Bae, and K. Belczynski, Mon. Not. R. Astron. Soc. 469, 4665 (2017).

[24] R. M. O’Leary, B. Kocsis, and A. Loeb, Mon. Not. R. Astron. Soc. 395, 2127 (2009).

[25] F. Antonini, S. Chatterjee, C. L. Rodriguez, M. Morscher, B. Pattabiraman, V. Kalogera, and F. A. Rasio, Astrophys. J. 816, 65 (2016).

[26] G. Nelemans, in The Astrophysics of Gravitational Wave Sources, American Institute of Physics Conference Series Vol. 686, edited by J. M. Centrella (AIP, New York, 2003), pp. 263-272.

[27] A. Sesana, J. Phys. Conf. Ser. 840, 012018 (2017).

[28] O. Blaes, M. H. Lee, and A. Socrates, Astrophys. J. 578, 775 (2002).

[29] L. Hoffman and A. Loeb, Mon. Not. R. Astron. Soc. 377, 957 (2007).

[30] P. Amaro-Seoane, A. Sesana, L. Hoffman, M. Benacquista, C. Eichhorn, J. Makino, and R. Spurzem, Mon. Not. R. Astron. Soc. 402, 2308 (2010).

[31] M. Bonetti, F. Haardt, A. Sesana, and E. Barausse, Mon. Not. R. Astron. Soc. 477, 3910 (2018).

[32] M. Bonetti, A. Sesana, F. Haardt, E. Barausse, and M. Colpi, Mon. Not. R. Astron. Soc. 486, 4044 (2019).

[33] T. Damour and N. Deruelle, Ann. Inst. Henri Poincaré Phys. Théor. 43, 107 (1985).

[34] R.-M. Memmesheimer, A. Gopakumar, and G. Schäfer, Phys. Rev. D 70, 104011 (2004).

[35] T. Damour, A. Gopakumar, and B. R. Iyer, Phys. Rev. D 70, 064028 (2004).

[36] C. Königsdörffer and A. Gopakumar, Phys. Rev. D 73, 124012 (2006).

[37] N. Yunes, K. G. Arun, E. Berti, and C. M. Will, Phys. Rev. D 80, 084001 (2009).

[38] N. J. Cornish and J.S. Key, Phys. Rev. D 82, 044028 (2010).

[39] J. S. Key and N. J. Cornish, Phys. Rev. D 83, 083001 (2011).
[40] E. A. Huerta, P. Kumar, S. T. McWilliams, R. O'Shaughnessy, and N. Yunes, Phys. Rev. D 90, 084016 (2014).

[41] E. A. Huerta, P. Kumar, B. Agarwal, D. George, H.-Y. Schive, H. P. Pfeiffer, R. Haas, W. Ren, T. Chu, M. Boyle, D. A. Hemberger, L. E. Kidder, M. A. Scheel, and B. Szilagyi, Phys. Rev. D 95, 024038 (2017).

[42] A. Gopakumar and G. Schäfer, Phys. Rev. D 84, 124007 (2011).

[43] S. Tanay, M. Haney, and A. Gopakumar, Phys. Rev. D 93, 064031 (2016).

[44] I. Hinder, L. E. Kidder, and H. P. Pfeiffer, Phys. Rev. D 98, 044015 (2018).

[45] Z. Cao and W.-B. Han, Phys. Rev. D 96, 044028 (2017).

[46] A. Klein, Y. Boetzel, A. Gopakumar, P. Jetzer, and L. de Vittori, Phys. Rev. D 98, 104043 (2018).

[47] Y. B. Zel'dovich and A. G. Polnarev, Sov. Astron. 18, 17 (1974).

[48] D. Christodoulou, Phys. Rev. Lett. 67, 1486 (1991).

[49] A. G. Wiseman and C. M. Will, Phys. Rev. D 44, R2945 (1991).

[50] K. S. Thorne, Phys. Rev. D 45, 520 (1992).

[51] L. Blanchet and T. Damour, Phys. Rev. D 46, 4304 (1992).

[52] P. N. Payne, Phys. Rev. D 28, 1894 (1983).

[53] A. Strominger and A. Zhiboedov, J. High Energy Phys. 16 (2016) 86.

[54] S. Pasterski, A. Strominger, and A. Zhiboedov, J. High Energy Phys. 16 (2016) 53.

[55] N. Seto, Mon. Not. R. Astron. Soc. 400, L38 (2009).

[56] R. van Haasteren and Y. Levin, Mon. Not. R. Astron. Soc. 401, 2372 (2010).

[57] J. M. Cordes and F. A. Jenet, Astrophys. J. 752, 54 (2012).

[58] J. B. Wang, G. Hobbs, W. Coles, R. M. Shannon, X. J. Zhu et al., Mon. Not. R. Astron. Soc. 446, 1657 (2015).

[59] M. Favata, Astrophys. J. 696, L159 (2009).

[60] P. D. Lasky, E. Thrane, Y. Levin, J. Blackman, and Y. Chen, Phys. Rev. Lett. 117, 061102 (2016).

[61] L. O. McNeill, E. Thrane, and P. D. Lasky, Phys. Rev. Lett. 118, 181103 (2017).

[62] M. Favata, Phys. Rev. D 80, 024002 (2009).

[63] M. Favata, Phys. Rev. D 84, 124013 (2011).

[64] L. Blanchet, G. Faye, B. R. Iyer, and S. Sinha, Classical Quantum Gravity 25, 165003 (2008).

[65] C. K. Mishra, K. G. Arun, and B. R. Iyer, Phys. Rev. D 91, 084040 (2015).

[66] Y. Boetzel, C. K. Mishra, G. Faye, A. Gopakumar, and B. R. Iyer, Phys. Rev. D 100, 044018 (2019).

[67] See Supplemental Material at http://link.aps.org/ supplemental/10.1103/PhysRevD.100.084043 for a Mathematica Notebook containing all memory modes presented in this manuscript. It also lists full expressions for all modes including instantaneous, hereditary and postadiabatic contributions.

[68] D. A. Nichols, Phys. Rev. D 95, 084048 (2017).

[69] L. Blanchet, Proc. R. Soc. Ser. A 409, 383 (1987).

[70] DLMF, NIST Digital Library of Mathematical Functions, edited by F. W. J. Olver, A. B. O. Daalhuis, D. W. Lozier, B. I. Schneider, R. F. Boisvert, C. W. Clark, B. R. Miller, and B. V. Saunders (2019), Release 1.0.22 of 2019-03-15, http://dlmf.nist.gov/. 
[71] Y. Boetzel, A. Susobhanan, A. Gopakumar, A. Klein, and P. Jetzer, Phys. Rev. D 96, 044011 (2017).

[72] L. Blanchet, B. R. Iyer, C. M. Will, and A. G. Wiseman, Classical Quantum Gravity 13, 575 (1996).

[73] K. G. Arun, L. Blanchet, B. R. Iyer, and M. S. S. Qusailah, Classical Quantum Gravity 21, 3771 (2004).

[74] P. C. Peters, Phys. Rev. 136, B1224 (1964).

[75] L. E. Kidder, Phys. Rev. D 77, 044016 (2008).

[76] G. Faye, S. Marsat, L. Blanchet, and B. R. Iyer, Classical Quantum Gravity 29, 175004 (2012).
[77] R. A. Isaacson, Phys. Rev. 166, 1272 (1968).

[78] K. G. Arun, L. Blanchet, B. R. Iyer, and M. S. S. Qusailah, Phys. Rev. D 77, 064034 (2008).

[79] K. G. Arun, L. Blanchet, B. R. Iyer, and M. S. S. Qusailah, Phys. Rev. D 77, 064035 (2008).

[80] K. G. Arun, L. Blanchet, B. R. Iyer, and S. Sinha, Phys. Rev. D 80, 124018 (2009).

[81] G. Faye, L. Blanchet, and B. R. Iyer, Classical Quantum Gravity 32, 045016 (2015). 